\title{
Secondary instability and tertiary states in rotating plane Couette flow
}

\author{
C. A. Daly ${ }^{1} \uparrow$, Tobias M. Schneider ${ }^{2,3}$, Philipp Schlatter ${ }^{4}$ and N. Peake ${ }^{1}$ \\ ${ }^{1}$ Department of Applied Mathematics and Theoretical Physics, Centre for Mathematical Sciences, \\ University of Cambridge, Wilberforce Road, Cambridge CB3 OWA, UK \\ ${ }^{2}$ Max Planck Institute for Dynamics and Self-Organization, Am Fassberg 17, \\ D-37077 Göttingen, Germany \\ ${ }^{3}$ ECPS, École Polytechnique Fédérale de Lausanne, 1015 Lausanne, Switzerland \\ ${ }^{4}$ Linné FLOW Centre, KTH Mechanics, Royal Institute of Technology, SE-100 44 Stockholm, Sweden
}

(Received 29 January 2014; revised 8 September 2014; accepted 15 October 2014; first published online 14 November 2014)

Recent experimental studies have shown rich transition behaviour in rotating plane Couette flow (RPCF). In this paper we study the transition in supercritical RPCF theoretically by determination of equilibrium and periodic orbit tertiary states via Floquet analysis on secondary Taylor vortex solutions. Two new tertiary states are discovered which we name oscillatory wavy vortex flow (oWVF) and skewed vortex flow (SVF). We present the bifurcation routes and stability properties of these new tertiary states and, in addition, we describe a bifurcation procedure whereby a set of defected wavy twist vortices is approached. Further to this, transition scenarios at flow parameters relevant to experimental works are investigated by computation of the set of stable attractors which exist on a large domain. The physically observed flow states are shown to share features with states in our set of attractors.

Key words: bifurcation, nonlinear instability, transition to turbulence

\section{Introduction}

The seminal work of Taylor (1923) mixed experiment and theory to describe the emergence of vortex structures in a differentially rotated concentric-cylinder apparatus. These vortex structures are now called Taylor vortices and are considered key structures in transition of cylindrical and curved fluid flow (see Koschmieder 1993 for a full review). The Taylor vortices were shown to develop due to a linear inviscid instability of the base flow, putting experiment and theory in good agreement with one another and providing some hope that the phenomena of transition and turbulence may be theoretically understood. Taylor's experiments sparked a flurry of research activity, which includes the following: theoretical studies of Taylor vortex instability such as Davey, DiPrima \& Stuart (1968) and Eagles (1971), who used weakly nonlinear analysis to determine the instabilities which affect Taylor vortices; experimental papers such as Coles (1965), Andereck, Liu \& Swinney (1986) and Hegseth, Baxter \& Andereck (1996), who mapped in parameter space the different flow regimes 
observed in the experiments; and numerical stability analyses wherein finite-amplitude Taylor vortices are calculated numerically in addition to the higher-order structures they bifurcate towards, such as Nagata (1988), Weisshaar, Busse \& Nagata (1991) and Antonijoan \& Sánchez (2000). Recent experimental attention has been focused on the flow of a differentially rotated fluid through a linear shear layer, known as rotating plane Couette flow (RPCF), a review of which can be found in Mullin (2010). Alfredsson \& Tillmark (2005), Hiwatashi et al. (2007), Tsukahara, Tillmark \& Alfredsson (2010), Suryadi, Tillmark \& Alfredsson (2013) and Suryadi, Segalini \& Alfredsson (2014) carried out experimental investigations of transition, with Tsukahara et al. (2010) following Andereck et al. (1986) by making a map in parameter space demarcating the different flow regimes existing. Though RPCF is a more challenging flow to study experimentally, it is perhaps more amenable to a theoretical or numerical analysis due to the Cartesian geometry and the ease with which rotation can be added to the governing equations. The equations governing RPCF can be interpreted as a local approximation to Taylor-Couette flow in the co-rotating, narrow-gap limit, and indeed many of the aforementioned theoretical and numerical studies are based in the Cartesian framework.

Theoretical progress in the understanding of transition in subcritical shear flows has included the discovery of exact coherent structures: finite-amplitude, spatially periodic numerical solutions to the Navier-Stokes equations. A body of work has grown detailing the properties of the structures in plane Couette flow (PCF): Nagata (1990), Clever \& Busse (1997), Nagata (1997), Waleffe (1997, 1998, 2003), Viswanath (2007), Gibson, Halcrow \& Cvitanović (2008), Gibson, Halcrow \& Cvitanović (2009), Itano \& Generalis (2009); and in pipe flow: Faisst \& Eckhardt (2003), Wedin \& Kerswell (2004), Pringle \& Kerswell (2007), Wang, Gibson \& Waleffe (2007) and Duguet, Pringle \& Kerswell (2008). Exact coherent structures have been shown to support chaotic dynamics (Gibson et al. 2009; Kreilos \& Eckhardt 2012), and also act as edge states between laminar and turbulent regimes (Schneider et al. 2008). Therefore, exact coherent structures play a key role in transition of the subcritical shear flows. It has been shown by Nagata (2013) that some exact coherent structures can be interpreted as having their origin as tertiary states in supercritical flows. We are thus motivated to explore the extent to which transitional dynamics in supercritical RPCF can be understood via the tertiary states supported by the flow.

The aims of this paper are twofold: we examine the inventory of tertiary states in RPCF via secondary stability analysis; and we investigate the extent to which such tertiary states inform the dynamics observed in physical experiments. We begin with a short review of the linear instabilities which destabilize the basic primary flow and the streamwise-independent finite-amplitude Taylor vortices, often called secondary states in this context, which emerge from each unstable mode. We perform a thorough investigation of the stability properties of Taylor vortices using Floquet theory, and use Taylor vortex instabilities as a starting point from which we can determine finite-amplitude tertiary states. This includes stability analysis of Taylor vortices with a range of spanwise wavenumbers $\beta$, to extend previous works such as Nagata (1998) who focused primarily on Taylor vortices in RPCF with the critical primary wavenumber, $\beta=1.5585$ in our non-dimensionalization. Our approach uncovers a new streamwise-independent structure and demonstrates that time-periodic states appear at moderate Reynolds numbers. We recover the tertiary states found by previous authors in their investigations of cylindrical Taylor-Couette flow, such as 
wavy vortex flow (Davey et al. 1968; Nagata 1988) and twist vortices (Weisshaar et al. 1991; Antonijoan \& Sánchez 2000). Our analysis of the bifurcation sequence in RPCF is subsequently used to study the laminar flow regimes observed in the experiments of Tsukahara et al. (2010) and Suryadi et al. (2014). Transition is addressed by accounting for the attractor states, i.e. stable secondary/tertiary states, which bifurcate via the first Taylor vortex solution, at parameter values corresponding to the experimental cases. In all cases considered we find a multiplicity of stable attractors, in agreement with the experimental observations of Benjamin \& Mullin (1982) for cylindrical Taylor-Couette flow. We compare features of our attractor states with the experimental results of Tsukahara et al. (2010) and Suryadi et al. (2014). Further to this, we perform a large-domain numerical simulation which reveals an additional attractor state, which takes the form of a localized fold amongst an array of Taylor vortices.

The structure of the paper is as follows: the governing equations and the geometry of the problem are introduced in $\S 2$ and we review the linear stability properties of the basic flow. In $\S 3$ an account is given of the methods we use to find nonlinear numerical secondary and tertiary solutions to the governing equations. In $\S 4$ we describe the Floquet theory techniques which we use to determine the stability of spatially and temporally periodic states. Section 5 contains a discussion of the streamwise-independent secondary states which bifurcate from the base flow, and in $\S 6$ we perform a linear Floquet stability analysis of the first Taylor vortex solution. Tertiary states are introduced in $\S 7$ and bifurcations of new nonlinear tertiary solutions are presented in $\S \S 8-10$. Finally, in $\S 11$ we investigate the relevance of the secondary and tertiary states to the transition phenomena observed in physical experiments of RPCF.

\section{Governing equations}

Rotating plane Couette flow is the flow of an incompressible Newtonian fluid under linear shear and Coriolis rotation, as shown in figure 1. The fluid is constrained between moving impermeable walls, with velocity difference $2 \tilde{U}$ between the upper and lower walls and channel width $2 \tilde{h}$. We define unit vectors $\boldsymbol{i}, \boldsymbol{j}$ and $\boldsymbol{k}$ pointing in the streamwise, wall-normal and spanwise directions $(x, y, z)$ respectively, and non-dimensionalize lengths by $\tilde{h}$, time by $\tilde{h} / \tilde{U}$ and pressure by $\tilde{U}^{2}$. With $\tilde{v}$ denoting the kinematic viscosity, we introduce three non-dimensional parameters: the Reynolds number $R e$, the rotation number $R o$ and a second rotation number $\Omega$,

$$
R e=\frac{\tilde{U} \tilde{h}}{\tilde{v}}, \quad R o=\frac{2 \tilde{\Omega} \tilde{h}}{\tilde{U}}, \quad \Omega=\frac{2 \tilde{\Omega} \tilde{h}^{2}}{\tilde{v}}=\operatorname{Re} R o .
$$

Any two of these non-dimensional parameters are independent. The nondimensionalized fluid velocity and pressure $(\overline{\boldsymbol{u}}, \bar{p})$ satisfy

$$
\begin{gathered}
\frac{\partial \overline{\boldsymbol{u}}}{\partial t}+\overline{\boldsymbol{u}} \cdot \nabla \overline{\boldsymbol{u}}=-\nabla \bar{p}+\frac{1}{R e} \nabla^{2} \overline{\boldsymbol{u}}+\operatorname{Ro}(\overline{\boldsymbol{u}} \times \boldsymbol{k}), \\
\nabla \cdot \overline{\boldsymbol{u}}=0,
\end{gathered}
$$

with the no-slip boundary condition imposed on the walls $y= \pm 1$. The linear shear profile $\boldsymbol{U}=y \boldsymbol{i}$ is a solution to this system with pressure distribution $P=1-\left(\operatorname{Ro} y^{2}\right) / 2$. Of the two rotation numbers $R o$ and $\Omega, R o$ is useful since it appears as a single 


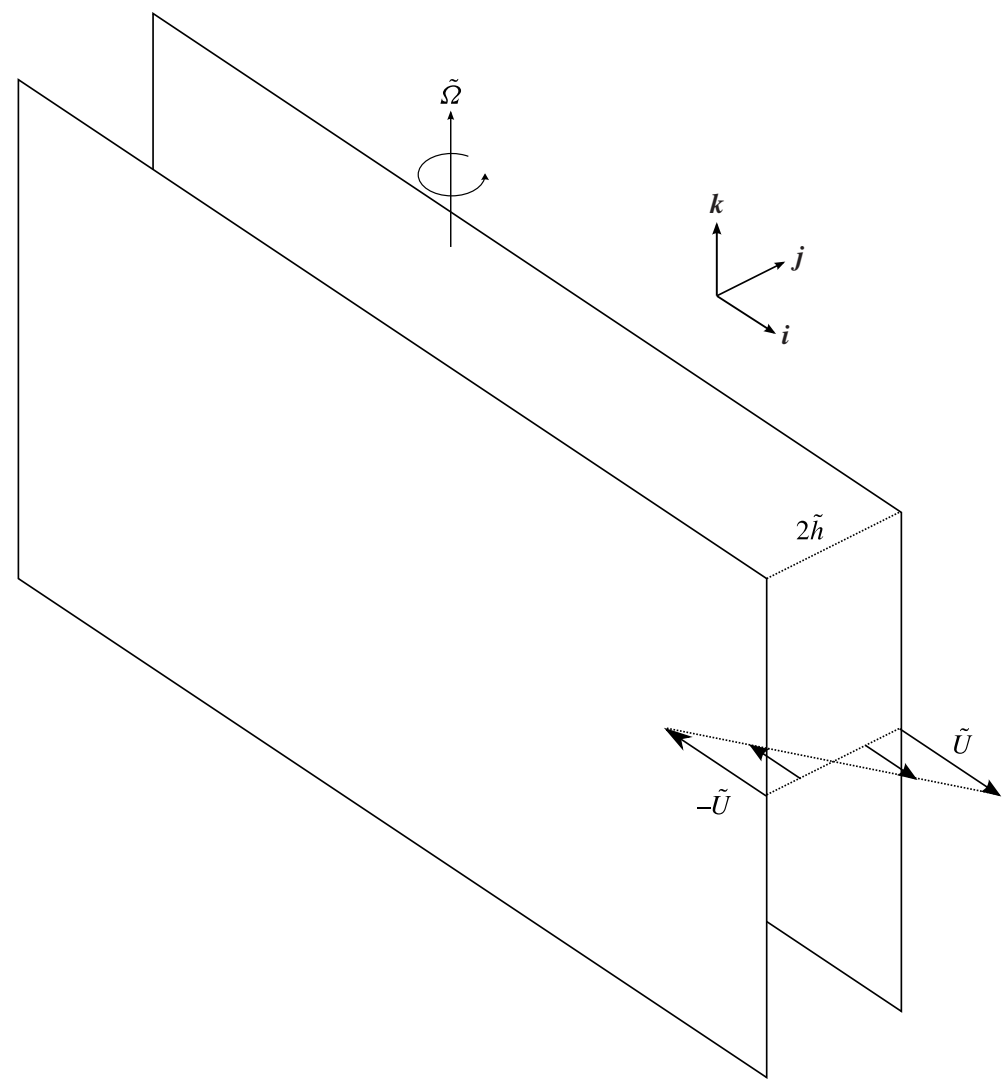

FIGURE 1. Rotating plane Couette flow geometry.

coefficient controlling the Coriolis force in (2.2), whereas $\Omega$ is independent of the wall velocity in terms of its non-dimensionalization, meaning that it describes the effects of rotation independently of $R e$. For the majority of this paper we describe rotation in terms of $R o$; however, in $\S 11$ we use $\Omega$, in accordance with experimental preference.

Lezius \& Johnston (1976) have shown that RPCF loses stability in the rotation parameter range $0<R o<1$, to spanwise-periodic disturbances with critical wavenumber $\beta \approx 1.5585$. The neutral curve takes the form

$$
R e^{2}=\frac{107}{R o(1-R o)} .
$$

Above the critical Reynolds number $R e_{c r} \approx 20.7$, there is a finite range of $R o$ for which the flow is linearly unstable. Solving the linear stability equations using the pseudo-spectral technique summarized in Schmid \& Henningson (2001) shows that linearly unstable perturbations take the form of either streamwise-oriented vortices, with streamwise wavenumber $\alpha=0$ and spanwise wavenumber $\beta$ non-zero, or obliquely oriented vortices with both $\alpha$ and $\beta$ non-zero. From the linear stability calculations we find that for fixed $\beta, R e$ and $R o$, the leading streamwise vortex eigenmode is generically more unstable than the leading oblique vortex eigenmode. We are therefore led to investigate the streamwise-independent secondary structures 
which bifurcate from the primary shear flow, since an arbitrary perturbation to the primary flow is most likely to visit a streamwise structure.

\section{Nonlinear states}

We refer to numerical solutions of the nonlinear equations governing RPCF as nonlinear states. For a given initial guess, a Newton-Krylov-hookstep algorithm as outlined in Viswanath (2007) is used to find a steady or time-periodic state. Typically, our initial guesses come from predictions given by linear stability theory. Calculations are made using the open-source channelflow software system (Gibson 2008), which has been modified to include the Coriolis terms. Numerical integration in channelflow is performed via spectral discretization in the periodic directions, and expansion of the wall-normal direction in Chebyshev polynomials. A semi-implicit backward differentiation technique is used to step the equations in time, and the nonlinearity is treated in rotational form. The pressure is solved for and updated at each time step, and the divergence-free criterion is maintained throughout. The Newton-Krylov-Hookstep algorithm, along with a solution continuation routine, is contained in the channelflow libraries.

Using the perturbation velocity $\boldsymbol{u}=\overline{\boldsymbol{u}}-\boldsymbol{U}$, we denote the perturbation Navier-Stokes equations for RPCF as

$$
\begin{gathered}
\frac{\partial \boldsymbol{u}}{\partial t}=L_{N S} \boldsymbol{u}, \\
\nabla \cdot \boldsymbol{u}=0,
\end{gathered}
$$

where

$$
L_{N S} \boldsymbol{u}=\frac{1}{R e} \nabla^{2} \boldsymbol{u}-\nabla p+R o(\boldsymbol{u} \times \boldsymbol{k})-\boldsymbol{U} \cdot \nabla \boldsymbol{u}-\boldsymbol{u} \cdot \nabla \boldsymbol{U}-\frac{1}{2} \nabla \boldsymbol{u}^{2}+\boldsymbol{u} \times(\nabla \times \boldsymbol{u}) .
$$

The time- $t$ forward map is given as

$$
F_{N S}^{t}(\boldsymbol{u})=\boldsymbol{u}+\int_{0}^{t} L_{N S} \boldsymbol{u}(\tau) \mathrm{d} \tau .
$$

We search for equilibrium solutions which satisfy

$$
\forall T>0, \quad F_{N S}^{T}(\boldsymbol{u})=\boldsymbol{u},
$$

and periodic orbits, for which

$$
\exists T>0, \quad F_{N S}^{T}(\boldsymbol{u})=\boldsymbol{u},
$$

where $T$, the period of a periodic orbit, is an unknown to be solved for. The NewtonKrylov-hookstep algorithm finds solutions by minimizing the residual $G(\boldsymbol{u})$ :

$$
G(\boldsymbol{u})=F_{N S}^{T}(\boldsymbol{u})-\boldsymbol{u} .
$$

The algorithm has been used by other authors (Viswanath 2007; Gibson et al. 2009) to find solutions in PCF, where the absence of linear instabilities makes finding an initial condition that converges towards a solution difficult. Though the basins of attraction of solutions in our case may generally be greater than in non-rotating plane Couette 
flow, due to supercriticality, the algorithm is still useful for its computational efficiency. All solutions found in this paper have an accuracy of $\|G(\boldsymbol{u})\|<10^{-10}$. The Coriolis terms in the equations of RPCF (3.1) do not break the symmetries satisfied by PCF. Thus RPCF, like PCF, is equivariant under the symmetry group $\Gamma$, which consists of rotation and translation operations. More detail on this symmetry group is given in appendix A.

Having found a state, we use the cross-flow energy $E_{c f}$ as a scalar measure of the nonlinear solution,

$$
E_{c f}\left(\alpha_{s}, \beta_{s}, R e, R o\right)=\frac{1}{2 \mathscr{D}_{V}} \int_{\mathscr{D}}\left(v^{2}+w^{2}\right) \mathrm{d} V,
$$

where $\mathscr{D}$ is the $x$ - and $z$-periodic domain $\left[0, L_{x}\right] \times[-1,1] \times\left[0, L_{z}\right]=\left[0,(2 \pi) / \alpha_{s}\right] \times$ $[-1,1] \times\left[0,(2 \pi) / \beta_{s}\right]$, and $\mathscr{D}_{V}$ is its volume. $E_{c f}$ is defined in this way so that it is independent of the domain. For example, no distinction is made between a given solution and the same solution on a doubled domain. The four-parameter dependence of $E_{c f}$ invites us to fix three parameters and investigate trajectories of solutions on a low-dimensional subspace. As such, we typically fix the geometric parameters $\left(\alpha_{s}, \beta_{s}\right)$ and investigate bifurcations in $R o$, with the remaining dynamic parameter $R e$ fixed.

\section{Floquet stability analysis}

Once we have found a spatio-temporally periodic nonlinear Navier-Stokes solution, its stability properties can be determined through Floquet analysis. Floquet theory is the most appropriate way to determine the stability of a spatio-temporally periodic solution over a range of detuning wavenumbers, which we will perform repeatedly throughout this paper. For example, to determine the stability of a state to perturbations subharmonic in $x$ via the Arnoldi method, the state must first be doubled in the $x$ direction. Repeating this process to determine the stability of a state over a range of detuned wavenumbers can become computationally expensive. Let $\overline{\boldsymbol{U}}_{s}(x, y, z, t)$ be such a solution, with $\overline{\boldsymbol{U}}_{s}$ denoting the full velocity field $\overline{\boldsymbol{U}}_{s}=\boldsymbol{U}+\boldsymbol{U}_{s}$, where $\boldsymbol{U}$ is the Couette base flow and $\boldsymbol{U}_{s}$ is a nonlinear perturbation. Given wavenumbers $\alpha_{s}$ in $x$ and $\beta_{s}$ in $z$ we express $\overline{\boldsymbol{U}}_{s}$ as a Fourier series in the form

$$
\overline{\boldsymbol{U}}_{s}(x, y, z, t)=\sum_{j=-\infty}^{\infty} \sum_{k=-\infty}^{\infty} \overline{\boldsymbol{U}}_{j k}(y, t) \exp \left(\mathrm{i}\left(j \alpha_{s} x+k \beta_{s} z\right)\right),
$$

where

$$
\overline{\boldsymbol{U}}_{j k}(y, t)=\frac{\alpha_{s} \beta_{s}}{4 \pi^{2}} \int_{0}^{2 \pi / \alpha_{s}} \int_{0}^{2 \pi / \beta_{s}} \overline{\boldsymbol{U}}_{s}(x, y, z, t) \exp \left(-\mathrm{i}\left(j \alpha_{s} x+k \beta_{s} z\right)\right) \mathrm{d} x \mathrm{~d} z .
$$

Since the perturbation velocity is divergence free, it can be written as the sum of the poloidal potential $\phi$ and the toroidal potential $\psi$ :

$$
\boldsymbol{u}=\boldsymbol{\nabla} \times \nabla \times \phi \boldsymbol{j}+\nabla \times \psi \boldsymbol{j} .
$$

The no-slip boundary conditions impose

$$
\phi=\phi^{\prime}=\psi=0,
$$


on the walls $y= \pm 1$. We apply the operators $j \cdot \nabla \times \nabla \times$ and $j \cdot \nabla \times$ to the linearized perturbation equations about $\overline{\boldsymbol{U}}_{s}$, to form two independent equations for $\phi$ and $\psi$ :

$$
\begin{gathered}
\partial_{t} \nabla^{2} \Delta_{2} \phi=\frac{1}{R e} \nabla^{4} \Delta_{2} \phi-R o \partial_{z} \Delta_{2} \psi-\boldsymbol{j} \cdot\left(\nabla \times \nabla \times\left(\boldsymbol{u} \cdot \nabla \overline{\boldsymbol{U}}_{s}+\overline{\boldsymbol{U}}_{s} \cdot \nabla \boldsymbol{u}\right)\right), \\
\partial_{t} \Delta_{2} \psi=\frac{1}{\operatorname{Re}} \nabla^{2} \Delta_{2} \psi+\operatorname{Ro} \partial_{z} \Delta_{2} \phi-\boldsymbol{j} \cdot\left(\nabla \times\left(\boldsymbol{u} \cdot \nabla \overline{\boldsymbol{U}}_{s}+\overline{\boldsymbol{U}}_{s} \cdot \nabla \boldsymbol{u}\right)\right),
\end{gathered}
$$

where $\Delta_{2}=\partial_{x}^{2}+\partial_{z}^{2}$ is the two-dimensional Laplacian and $\nabla^{2}=\partial_{x}^{2}+\partial_{y}^{2}+\partial_{z}^{2}$ is the three-dimensional Laplacian. Following from continuity and the boundary conditions at $y= \pm 1$, the mean flow has no wall-normal component:

$$
\boldsymbol{U}_{00}=U(y, t) \boldsymbol{i}+W(y, t) \boldsymbol{k} .
$$

The velocity perturbation $\boldsymbol{u}$ in system (4.5) is mapped to the poloidal-toroidal potentials, $\boldsymbol{\xi}=(\phi, \psi)$, using (4.3). It is convenient to write the system (4.5) more succinctly using operator notation:

$$
\begin{gathered}
\partial_{t} M \boldsymbol{\xi}=L\left(\overline{\boldsymbol{U}}_{s}\right) \boldsymbol{\xi} \\
\Longrightarrow \partial_{t} \boldsymbol{\xi}=\mathscr{L}\left(\overline{\boldsymbol{U}}_{s}\right) \boldsymbol{\xi}, \quad \mathscr{L}\left(\overline{\boldsymbol{U}}_{s}\right)=M^{-1} L\left(\overline{\boldsymbol{U}}_{s}\right) .
\end{gathered}
$$

Since $\overline{\boldsymbol{U}}_{s}$ is periodic in time so too is the operator $\mathscr{L}\left(\overline{\boldsymbol{U}}_{s}\right)$. We now construct the Poincaré map, $P\left(\overline{\boldsymbol{U}}_{s}\right)$, which is formally given by

$$
P\left(\overline{\boldsymbol{U}}_{s}\right)=\exp \left(\int_{0}^{T} \mathscr{L}\left(\overline{\boldsymbol{U}}_{s}\right) \mathrm{d} t\right) .
$$

The Poincaré map gives the action of system (4.8) on an arbitrary potential vector $\boldsymbol{\xi}$ over one period, i.e.

$$
\boldsymbol{\xi}(\boldsymbol{x}, t+T)=P\left(\overline{\boldsymbol{U}}_{s}\right) \boldsymbol{\xi}(\boldsymbol{x}, t) .
$$

The eigenvalues $\mu$ of $P\left(\overline{\boldsymbol{U}}_{s}\right)$ are the Floquet multipliers over the period

$$
\mu=\mathrm{e}^{\sigma T},
$$

from which we determine the exponent $\sigma$.

En route to computing $P\left(\overline{\boldsymbol{U}}_{s}\right)$, Floquet's theorem implies that $\xi$ need not have the same periodicity as $\overline{\boldsymbol{U}}_{s}$, so we write

$$
\begin{gathered}
\xi(x, y, z, t)=\mathrm{e}^{\mathrm{i}(\alpha x+\beta z)+\sigma t} \sum_{m=-\infty}^{\infty} \sum_{n=-\infty}^{\infty} \hat{\boldsymbol{\xi}}_{m n}(y, t) \exp \left(\mathrm{i}\left(m \alpha_{s} x+n \beta_{s} z\right)\right), \\
0 \leqslant \alpha \leqslant \frac{\alpha_{s}}{2}, \\
0 \leqslant \beta \leqslant \frac{\beta_{s}}{2},
\end{gathered}
$$

where $\alpha$ and $\beta$ are the detuning parameters. The modes $\hat{\xi}_{m n}(y, t)$ are assumed to be periodic in time with the same period as $\overline{\boldsymbol{U}}_{s}$. Therefore we can frame (4.8) as a system of infinitely many coupled equations

$$
\partial_{t} P_{m n} \hat{\boldsymbol{\xi}}_{m n}=Q_{m n} \hat{\boldsymbol{\xi}}_{m n}+\sum_{j, k} F_{m n}^{j k} \hat{\boldsymbol{\xi}}_{m-j, n-k}, \quad \forall m, n \in \mathbb{Z},
$$


for operators $P_{m n}, Q_{m n}$ and $F_{m n}^{j k}$ given in appendix B. We truncate the system in the periodic directions with $m \leqslant\left|T_{m}\right|$ and $n \leqslant\left|T_{n}\right|$, and discretize in the wall-normal direction with $N$ Chebyshev polynomials. With the system suitably discretized and truncated, we use the second-order implicit trapezium rule method to advance the equations in time. The trapezium rule is chosen because we found that explicit methods were generally unstable and higher-order methods have greater computational memory requirements at each time step. The action of the Poincare map is found by time-stepping the equations over one period $T$. At each time step the state $\overline{\boldsymbol{U}}_{s}$ must be updated. We do this by Fourier series approximation of the base flow at the desired time point. From direct numerical simulation (DNS) of $\overline{\boldsymbol{U}}_{s}$ over one period we save the flowfield at 100 time points, and from these we numerically approximate the temporal Fourier transform

$$
\widetilde{\boldsymbol{U}}_{\ell}=\int_{0}^{T} \overline{\boldsymbol{U}}_{s}(t) \mathrm{e}^{(\mathrm{i} 2 \pi \ell t) / T} \mathrm{~d} t,
$$

so that the flowfield at an arbitrary time $t$ can be expressed by the Fourier series

$$
\overline{\boldsymbol{U}}_{s}(t)=\sum_{\ell=-\infty}^{\infty} \widetilde{\boldsymbol{U}}_{\ell} \mathrm{e}^{-(\mathrm{i} 2 \pi \ell t) / T} .
$$

A summation truncation of $-30 \leqslant \ell \leqslant 30$ is used. Comparison of the Fourier series approximation of the flowfield with a previously saved field has an error $O\left(10^{-11}\right)$.

The advantages of using the poloidal-toroidal potentials rather than primitive variables are that we do not need an extra calculation to update the pressure at each time step. The problem size is thus reduced by half relative to a primitive variables formulation, meaning that the operators are approximated by square matrices of order $M=2 N\left(2 T_{m}+1\right)\left(2 T_{n}+1\right)$. This eases the memory requirements for each stability calculation and allows higher truncations to be reached. Time-stepping of the linear perturbation equations and the final eigenvalue calculations are performed in MATLAB, while DNS of $\overline{\boldsymbol{U}}_{s}$ required for the temporal Fourier transform is carried out using channelflow (Gibson 2008). In the description above, we have used $\sigma$ as the perturbation growth rate. Throughout the rest of the paper, we adopt the convention of using $\omega, \sigma$ and $\tau$ to denote primary, secondary and tertiary instability complex frequencies, respectively.

\section{Streamwise-independent secondary states}

In this section we characterize the streamwise-independent secondary states in supercritical RPCF. We demonstrate the effect of rotation on the solutions by making bifurcation diagrams with bifurcation parameter $R o$ and using $E_{c f}$ as a measure of nonlinear states.

A bifurcation from the primary state initiated by the leading streamwise-dependent vortex instability eigenmode results in the so-called first Taylor vortex flow $\left(\mathrm{TVF}_{1}\right)$ (Nagata 1986; Koschmieder 1993). TVF 1 consists of a pair of streamwise-independent counter-rotating vortices and counter-propagating streaks, with an example flowfield shown in figure $2(a)$. The form of the flowfield is sensitive to changes in the spanwise periodicity of the state: both the streak and vortex components of the state are dependent upon the wavenumber of the state. For this reason we perform stability calculations across a range of wavenumbers in $\S 6 . \mathrm{TVF}_{1}$ flowfields are invariant in 
(a)

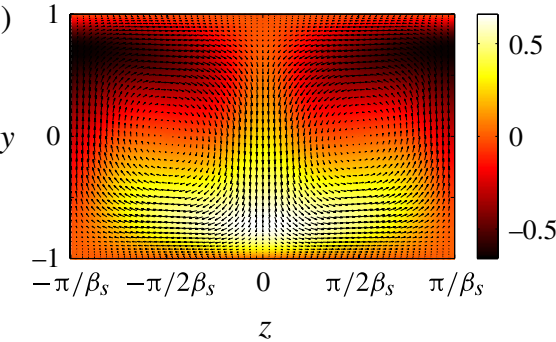

(b)

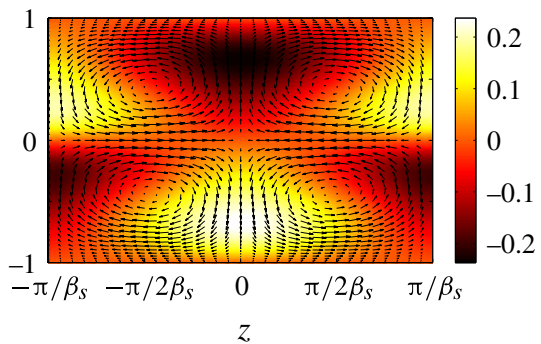

FIgURE 2. (Colour online) (a) First Taylor vortex flowfield $\left(\mathrm{TVF}_{1}\right)$, with $\left(\beta_{s}, R e, R o\right)=$ $(2,100,0.3)$. (b) Second Taylor vortex flowfield $\left(\mathrm{TVF}_{2}\right)$ with $\left(\beta_{s}, \operatorname{Re}, R o\right)=(2,100,0.5)$. Flowfields are depicted as colour plots of $u$ and vector plots of $(v, w)$ in the $(y, z)$-plane.

the streamwise direction, and as such they satisfy a continuous $x$-translation symmetry $\boldsymbol{T}\left(\ell_{x}\right)$, alongside discrete mirror symmetry $\boldsymbol{Z}$ in the $(y, z)$-plane:

$$
\begin{gathered}
\boldsymbol{T}\left(\ell_{x}\right)[u, v, w](x, y, z)=[u, v, w]\left(x+\ell_{x}, y, z\right), \\
\boldsymbol{Z}[u, v, w](x, y, z)=[u, v,-w](x, y,-z) .
\end{gathered}
$$

The mirror symmetry feature is apparent from the flowfield in figure $2(a)$, with the axis of reflection being $z=0$ in the spanwise domain. The bifurcation in $R o$ of $\mathrm{TVF}_{1}$ from the laminar primary state (LAM) breaks the continuous spanwise symmetry of LAM, and is thus a supercritical continuous-symmetry-breaking (CSB) pitchfork bifurcation. The bifurcation is qualitatively independent of spanwise wavenumber, and a typical bifurcation of $\mathrm{TVF}_{1}$ from the primary state $\left(E_{c f}=0\right)$ is plotted in figure 3 .

As noted by Nagata (2013), the primary flow loses stability to a second streamwise vortex eigenmode, prompting a bifurcation to a second Taylor vortex flow $\left(\mathrm{TVF}_{2}\right)$. $\mathrm{TVF}_{2}$ has a double-layered vortical structure, with a pair of counter-rotating vortices aligned in the wall-normal direction, alongside another pair of counter-rotating vortices in the spanwise direction, as can be seen in figure $2(b)$. The symmetries $\boldsymbol{T}\left(\ell_{x}\right)$ and $\boldsymbol{Z}$ are also held by $\mathrm{TVF}_{2}$, with the axis of reflection $z=0$ for mirror symmetry in the spanwise domain of our figures. Bifurcations of $\mathrm{TVF}_{2}$ in $R o$ are plotted in figure 3. The shape of the solution branch is reminiscent of those of $\mathrm{TVF}_{1}$ under bifurcation in $R o$, emerging in a CSB pitchfork bifurcation from $\mathrm{LAM}^{\mathrm{TV}} \mathrm{TVF}_{2}$ is unstable at each point in our bifurcation diagram.

\section{Stability of Taylor vortex flow}

In this section we determine the global stability of Taylor vortex flow. In particular, we focus on the stability of $\mathrm{TVF}_{1}$ : since the least-stable primary instability develops into this state, we anticipate that the secondary instabilities which emerge when $\mathrm{TVF}_{1}$ loses stability will have a prominent effect on any tertiary dynamics. Let $\beta_{s}$ denote the spanwise wavenumber of a $\mathrm{TVF}_{1}$ vortex pair. By varying the detuning parameters $\alpha$ and $\beta$ from (4.12) and solving the stability equation (4.13), we can determine the global stability of a Taylor vortex solution. It is typically found that the least-stable perturbations are either streamwise periodic and tuned to the fundamental spanwise wavenumber, i.e. $(\alpha, \beta)=(\alpha, 0)$, or of Eckhaus type with $(\alpha, \beta)=(0, \beta)$ (Eckhaus $1965)$. The wavenumber domain which spans the full stability properties is the semiinfinite strip

$$
0 \leqslant \alpha<\infty, \quad 0 \leqslant \beta \leqslant \frac{\beta_{s}}{2} .
$$




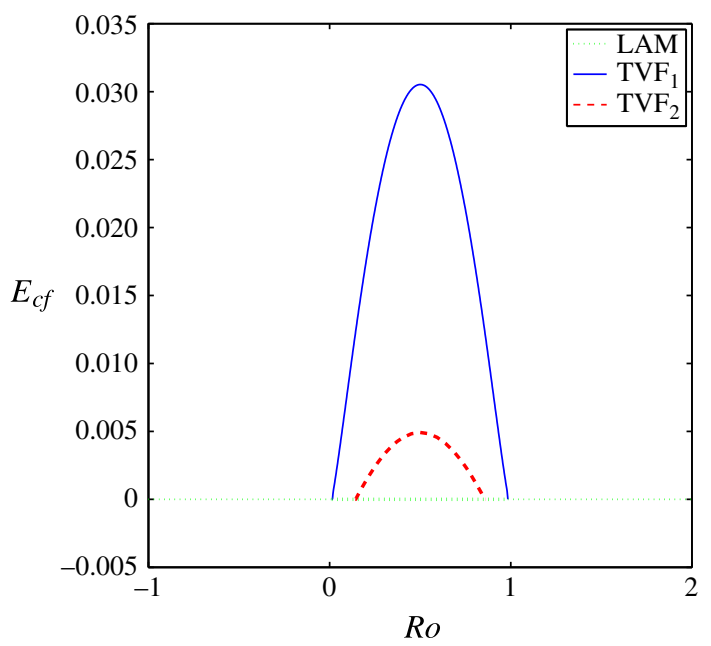

FIgURE 3. (Colour online) Bifurcation of $\mathrm{TVF}_{1}$ and $\mathrm{TVF}_{2}$ from primary state LAM for $\left(\beta_{s}, R e\right)=(2,100) . \mathrm{TVF}_{1}$ is harmonically stable throughout the trajectory whereas $\mathrm{TVF}_{2}$ is harmonically unstable. Both $\mathrm{TVF}_{1}$ and $\mathrm{TVF}_{2}$ bifurcate in supercritical, continuous-symmetry-breaking (CSB) pitchfork bifurcations from LAM.

We choose Reynolds numbers $R e=50$ and $R e=100$ then calculate the streamwise and spanwise secondary instabilities at various $\beta_{s}$. The chosen Reynolds numbers fall within the transition region, as observed experimentally in Tsukahara et al. (2010). The spanwise wavenumbers of the Taylor vortices, $\beta_{s}$, are chosen such that they are spread across the linearly unstable region of the primary flow, as shown in figure 4 where the maximum primary instability growth rate $\omega_{r}=\operatorname{Re}\{\omega\}$ is plotted in the $(\beta, R o)$-plane. Previous studies (Nagata 1988; Weisshaar et al. 1991) consider spanwise wavenumbers close to linear-critical value $\beta_{s}=1.5585$; however, we find that the stability characteristics of $\mathrm{TVF}_{1}$ are considerably different for $\beta_{s}$ far from the linear-critical value.

\subsection{Streamwise instability}

In figure 5 we plot a selection of streamwise instabilities of $\mathrm{TVF}_{1}$ with $R e=50$. The instabilities are indicated by contours of positive secondary instability growth rate, $\sigma_{r}=\operatorname{Re}\{\sigma\}>0$. For $\beta_{s}=1,1.5,2$ and 2.5 we see a wedge-shaped region of instability for $0 \lesssim R o \lesssim 0.2$ and $0 \lesssim \alpha \lesssim 0.6$. This is known as wavy vortex instability (Davey et al. 1968; Nagata 1986, 1998), which causes Taylor vortices to lose stability to structures elongated in the streamwise direction, with modulated high-velocity/lowvelocity streaks. Throughout the wedge regions in figure 5, the stability operator has one unstable eigenmode with $\sigma_{i}=0$. As the spanwise wavenumber of $\mathrm{TVF}_{1}$ increases to $\beta_{s}=3$, the wavy instability is no longer present and the vortices become stable to all streamwise perturbations. For $\beta_{s}=1$ two overlapping regions of instability occur at high rotation parameters, $0.6 \lesssim R o \lesssim 1$ and $0.5 \lesssim \alpha \lesssim 1.5$. These are known as twist vortex and wavy twist vortex instabilities (Weisshaar et al. 1991). The twist instabilities are more sensitive to the spanwise wavelength of $\mathrm{TVF}_{1}$ than the wavy instability, as they are not observed for $\beta_{s} \geqslant 1.5$.

Raising $R e$ to 100 has the effect of increasing the range and strength of instabilities affecting $\mathrm{TVF}_{1}$, as shown in the stability maps for $R e=100$ in figure 6. The wavy 

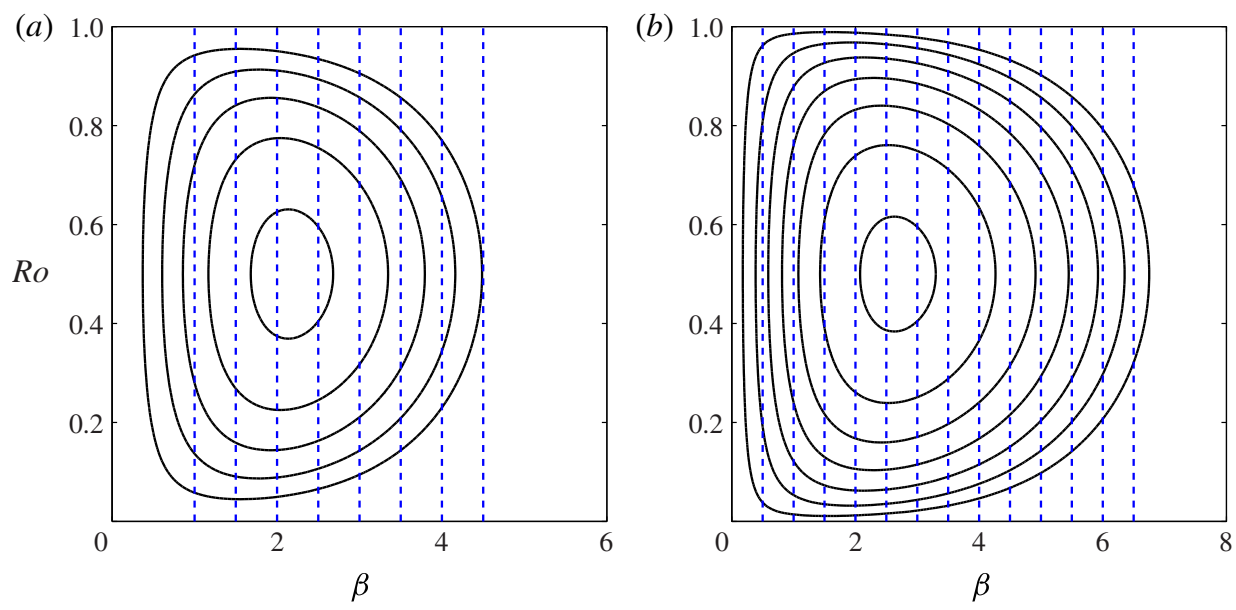

FIgURE 4. (Colour online) Contour plots of the maximum primary instability growth rate $\omega_{r} \geqslant 0$ against spanwise wavenumber $\beta$ and $R o$, at $(a) R e=50$ and $(b) R e=100$. In each plot the contours increase inwards from 0 in steps of 0.05 . The dashed lines mark the spanwise wavenumbers $\beta_{s}$ for which we conduct stability analysis of $\mathrm{TVF}_{1} ; \mathrm{TVF}_{1}$ with $\left(\beta_{s}, R e\right)=(0.5,50)$ could not be found to satisfactory accuracy, and is omitted.

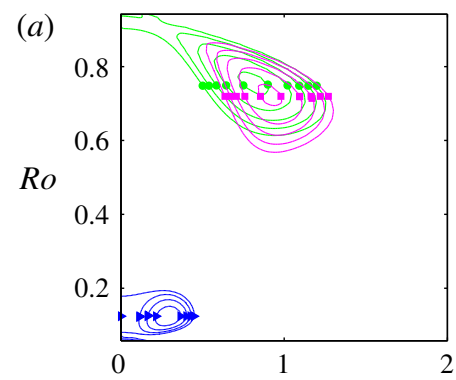

(b)
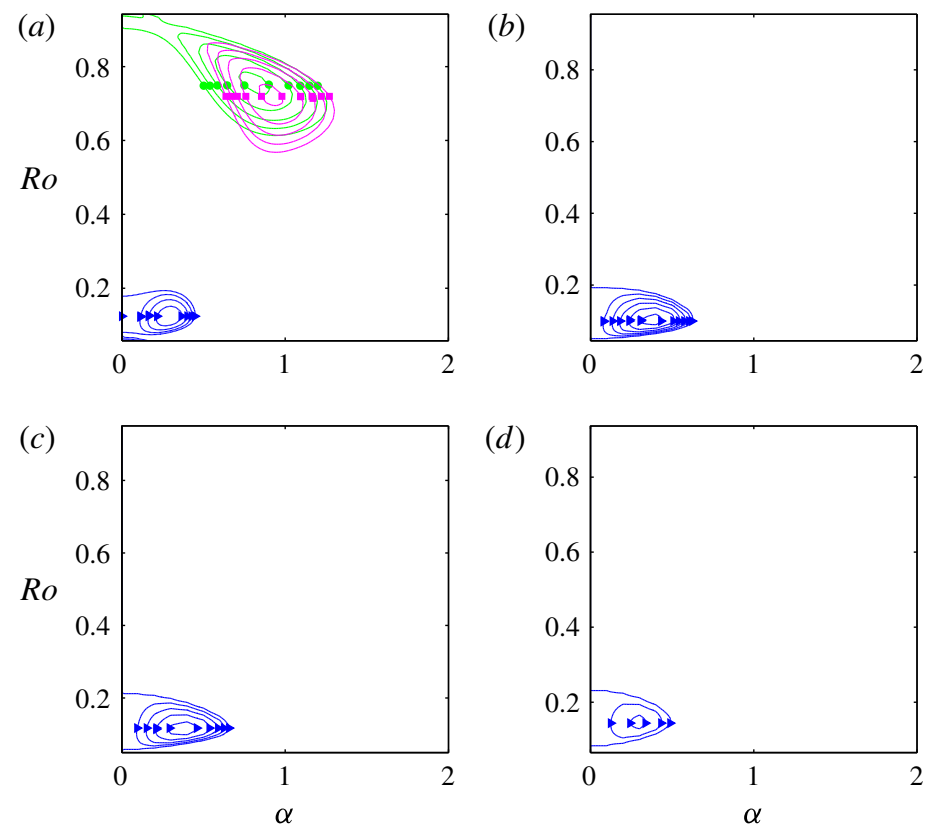

FIgURE 5. (Colour online) Streamwise instability for $R e=50$, and $\beta_{s}$ values: $(a) 1,(b)$ 1.5, (c) 2, (d) 2.5. Contours of $\sigma_{r} \geqslant 0$ are plotted in $(\alpha, R o)$-space, with contours increasing inwards from zero in intervals of 0.005 . Triangles (blue) $\equiv$ wavy instability, squares (magenta) $\equiv$ twist instability and circles (green) $\equiv$ wavy twist instability. High-Ro twist instabilities for $\beta_{s}=1$ are lost for $\beta_{s} \geqslant 1.5$. Low-Ro wavy instability persists through $\beta_{s}=$ $1, \ldots, 2.5$, and disappears for $\beta_{s} \geqslant 3$ as $\mathrm{TVF}_{1}$ becomes stable to streamwise perturbations. 
(a)

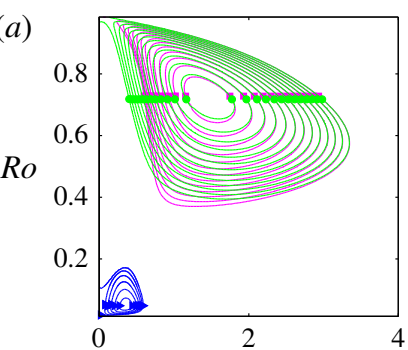

(c)

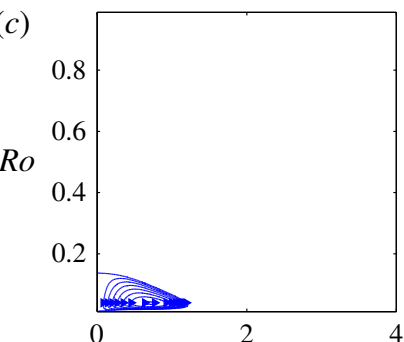

(e)

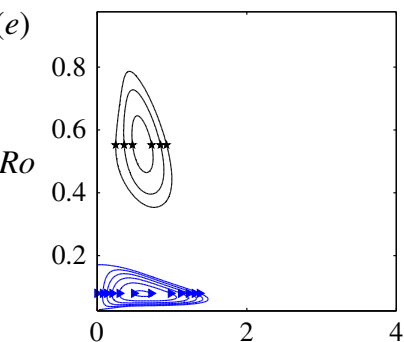

$(g)$

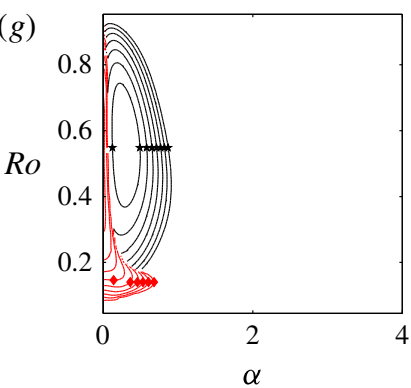

(b)

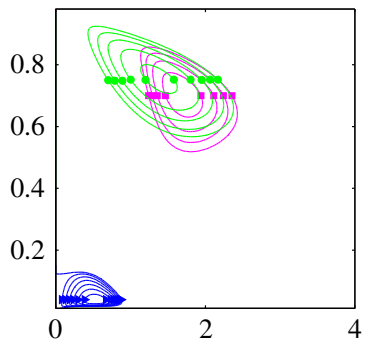

(d)

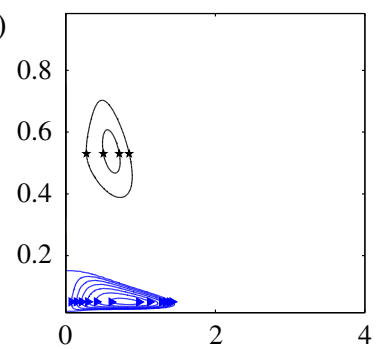

$(f)$

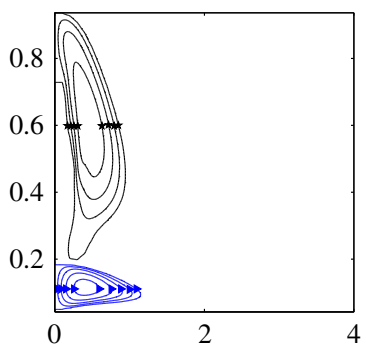

(h)

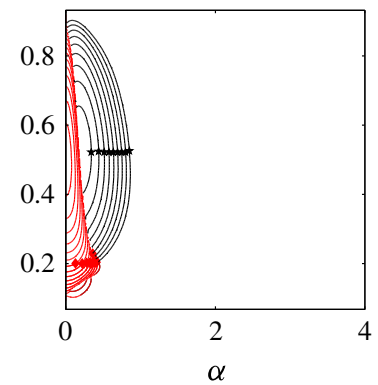

FIguRE 6. (Colour online) Streamwise instability for $R e=100$, and $\beta_{s}$ values: (a) 1, (b) 1.5, (c) 2, (d) 2.5, (e) 3, (f) 3.5, ( $g$ ) 4, (h) 4.5. Contours of $\sigma_{r} \geqslant 0$ are plotted in $(\alpha, R o)$-space, with contours increasing inwards from zero in intervals of 0.01 . Triangles (blue) $\equiv$ wavy instability, squares (magenta) $\equiv$ twist instability, circles (green) $\equiv$ wavy twist instability, stars (black) $\equiv$ oscillatory wavy instability and diamonds (red) $\equiv$ skew instability. High-Ro twist instabilities for $\beta_{s}=1,1.5$ are lost for $\beta_{s} \geqslant 2$. Oscillatory instability emerges for $\beta_{s}=2.5$ over a mid-Ro range, and strengthens as $\beta_{s}$ increases. Low-Ro wavy instability persists through $\beta_{s}=1, \ldots, 3.5$, before merging with the skew instability at $\beta_{s}=4$. 
vortex instability retains the wedge shape observed for $R e=50$, though the region stretches to $\alpha>1$ for $\beta_{s}=2.5,3$, and is confined to a smaller Ro range in comparison to $R e=50$. For $0.4 \lesssim R o \lesssim 1$ and $\beta_{s}=1,1.5$ we see a region containing both twist and wavy twist instabilities in figure 6 . The twist, wavy twist and wavy instabilities are also present in the stability maps of $\mathrm{TVF}_{1}$ with $\beta_{s}=0.5$ (not shown). A mid-Ro instability emerges for $\beta_{s}=2.5$ and strengthens as $\beta_{s}$ increases to 3.5 . The stability operator has a pair of complex-conjugate modes in this case so we term this the oscillatory wavy instability, since we expect unstable perturbations to oscillate in time as their amplitude grows. The instability first occurs in the range $0.5 \lesssim \alpha \lesssim 1$, giving the modes a streamwise-elongated appearance similar to the low-Ro wavy instability modes. This instability was noted by Nagata (1988), who has shown that for $\mathrm{TVF}_{1}$ with spanwise wavenumber $\beta_{s}=1.5585$, the instability first emerges for $\operatorname{Re} \approx 137.5$. As $\beta_{s}$ increases, the oscillatory instability is present over a widening range of $R o$ until it merges with the low-Ro wavy instability. This scenario is depicted in figure 6 for $\beta_{s}=4$ and $\beta_{s}=4.5$. Along the interface between the red and black contours denoted with diamonds and stars in the figure, the complex-conjugate oscillatory mode is split into two stationary modes. The upper mode (with larger $\sigma$ ) is denoted with diamonds (red) while the lower mode is not included in figure 6, since it is quickly stabilized as $\alpha$ approaches zero. The upper stationary mode creates a harmonic instability (with detuning parameters $\alpha=\beta=0$ ) appearing across the entire Ro range at which $\mathrm{TVF}_{1}\left(\beta_{s}=4\right)$ is unstable. Since this mode is stationary, but streamwise independent, we treat it as distinct from the streamwise-periodic wavy instability, though it can be interpreted as a deformation of the wavy instability brought about by the merger with the oscillatory mode. The harmonic instability persists as $\beta_{s}$ is increased to $\beta_{s}=4.5$. We find that the unstable harmonic mode causes the $\mathrm{TVF}_{1}$ vortex streaks to be tilted in the $(y, z)$-plane direction, therefore we call this the skew instability. The skewed effect can be seen in the skewed vortex flow (SVF) flowfields in figure 10. Streamwise instabilities disappear and $\mathrm{TVF}_{1}$ becomes globally stable for $\beta_{s} \geqslant 5$.

\subsection{Eckhaus instability}

An instability caused by a perturbation which is periodic in the same direction as the base state is called Eckhaus instability (Eckhaus 1965). For $R e=50, \mathrm{TVF}_{1}$ is stable to spanwise perturbations for $\beta_{s} \leqslant 2.5$. For $\beta_{s} \geqslant 3$, we find low-Ro and high-Ro tongues of subharmonic instability. An example for the case $\left(\beta_{s}, R e\right)=(3,50)$ is shown in figure $7(a)$. These unstable modes have $\sigma_{i}=0$, where $\sigma_{i}=\operatorname{Im}\{\sigma\}$, and initiate a bifurcation towards $\mathrm{TVF}_{1}$ with a lower $\beta_{s}$. For $R e=100$, we again find tongues of subharmonic instability for $\beta_{s}>3.5$, though the tongues are now restricted to a smaller range of high and low Ro. An additional region of instability emerges around the harmonic skew instability, an example of which for $\left(\beta_{s}, R e\right)=(4,100)$ is plotted in figure $7(b)$.

\section{Tertiary states of RPCF}

We define a tertiary state to be a structure which bifurcates directly from a secondary, but not the primary, state. In this analysis we identify a range of tertiary states which bifurcate from $\mathrm{TVF}_{1}$ at transitional Reynolds numbers. To assess the effects of rotation on the tertiary states we compute solution branches using $E_{c f}$ as a bifurcation parameter and present $R o$-bifurcation diagrams in $\S \S 8-10$. We use the Newton-Krylov-hookstep algorithm outlined in $\S 3$ to find the tertiary states based 

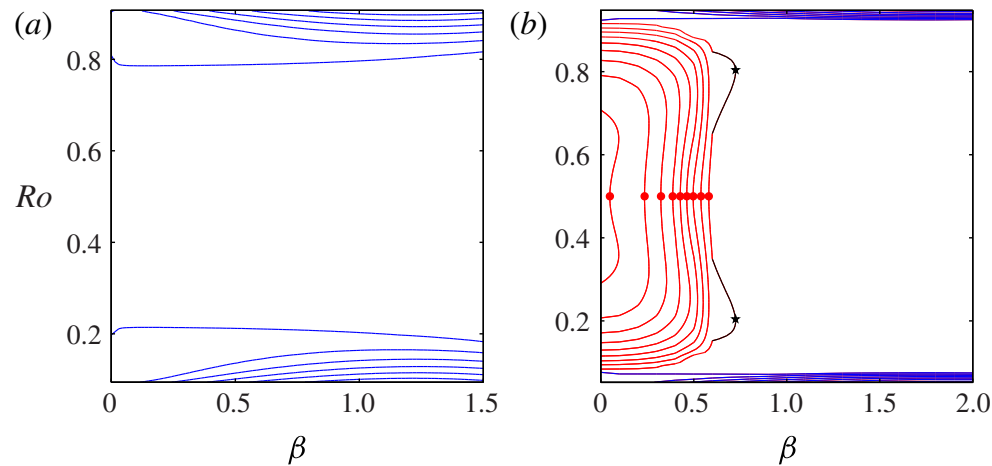

FIguRE 7. (Colour online) Eckhaus instability for $(a)\left(\alpha, \beta_{s}, R e\right)=(0,3,50)$ and $(b)$ $\left(\alpha, \beta_{s}, R e\right)=(0,4,100)$. Contours of $\sigma_{r} \geqslant 0$ are plotted in $(\beta, R o)$-space, with contours increasing inwards from zero in intervals of 0.01 . Plain line (blue) $\equiv$ subharmonic instability, dots (red) $\equiv$ skew instability and stars (black) $\equiv$ oscillatory instability. The skew and oscillatory instabilities here are special cases of the skew and oscillatory instabilities in figure 6 , with $\alpha=0$. The upper/lower tongues of subharmonic instability have contours increasing towards the upper/lower $R o$ boundaries. The harmonic instability for $\left(\alpha, \beta_{s}, R e\right)=(0,4,100)$ merges with an oscillatory instability for $\beta \approx 0.6,0.15<R o<$ 0.356 and $0.652<R o<0.844$.

on initial guesses from the Floquet theory predictions of $\S 6$. An adaptive quadratic continuation technique is then used to continue the states along the bifurcation parameter.

In table 1 we list the known tertiary states of supercritical RPCF along with their symmetry properties. We have included all the tertiary solutions discussed in this paper, and in addition the mirror-symmetric ribbon (RIB) equilibrium state which bifurcates from $\mathrm{TVF}_{2}$, as discussed by Nagata (2013). This inventory of tertiary states is almost certainly incomplete: the tertiary states which bifurcate from $\mathrm{TVF}_{2}$ are yet to be fully studied. Furthermore, there is a family of oblique secondary states, related to the spiral solutions of Taylor-Couette flow such as presented by Deguchi \& Altmeyer (2013), which we have omitted from this analysis.

We begin with an introduction to each of the tertiary states which bifurcate from $\mathrm{TVF}_{1}$. Wavy vortex flow (WVF) is a steady structure which develops when $\mathrm{TVF}_{1}$ loses stability to wavy vortex modes, with a flowfield consisting of counter-propagating streamwise-modulated vortex streaks. WVF is well-known from Taylor-Couette flow (see for example Davey et al. 1968; Andereck et al. 1986; Nagata 1986; Koschmieder $1993)$, and is discussed by Nagata $(1990,1998)$ in the context of RPCF. WVF breaks the mirror symmetry $\boldsymbol{Z}$ of $\mathrm{TVF}_{1}$, but holds the discrete shift-reflection and shiftrotation symmetries $\boldsymbol{S}$ and $\boldsymbol{\Omega}$ :

$$
\begin{gathered}
S[u, v, w](x, y, z)=[u, v,-w]\left(x+\frac{\pi}{\alpha}, y,-z+\frac{\pi}{\beta}\right), \\
\boldsymbol{\Omega}[u, v, w](x, y, z)=[-u,-v, w]\left(-x,-y, z+\frac{\pi}{\beta}\right) .
\end{gathered}
$$




\begin{tabular}{|c|c|c|c|c|}
\hline State & Bifurcation & Solution & Dimensionality & Symmetries \\
\hline $\mathrm{TVF}_{1}$ & CSB pitchfork/LAM & EQ & $2 \mathrm{D}$ & $\boldsymbol{T}\left(\ell_{x}\right), \boldsymbol{Z}$ \\
\hline $\mathrm{TVF}_{2}$ & CSB pitchfork/LAM & EQ & $2 \mathrm{D}$ & $\boldsymbol{T}\left(\ell_{x}\right), \boldsymbol{Z}$ \\
\hline WVF & DSB pitchfork/TVF 1 & EQ & $3 \mathrm{D}$ & $S, \Omega$ \\
\hline TWI & CSB pitchfork/TVF $\mathrm{TV}_{1}$ & EQ & $3 \mathrm{D}$ & $Z, \Omega$ \\
\hline wTWI & DSB pitchfork/TVF 1 & EQ & $3 \mathrm{D}$ & $S, \Omega$ \\
\hline oWVF & DSB Hopf/TVF & $\mathrm{PO}$ & $3 \mathrm{D}$ & $S, \Omega$ \\
\hline SVF & DSB pitchfork/(TVF $\left.F_{1}, \mathrm{TVF}_{2}\right)$ & EQ & $2 \mathrm{D}$ & $\boldsymbol{T}\left(\ell_{x}\right)$ \\
\hline RIB & CSB pitchfork $/ \mathrm{TVF}_{2}$ & EQ & $3 \mathrm{D}$ & $Z, S, \Omega$ \\
\hline
\end{tabular}

TABLE 1. Inventory of the secondary and tertiary states which bifurcate from the laminar state in RPCF. In the 'Bifurcation' column we report the type of bifurcation that the state makes, whether it is continuous-symmetry breaking (CSB) or discrete-symmetry breaking (DSB), followed by the state from which it bifurcates. The 'Solution' column indicates whether the solution is an equilibrium state (EQ) or periodic orbit (PO). The 'Dimensionality' of each state is meant in the sense of its $x$-dependence; we refer to $x$-invariant states as 2D. All states have 3D velocity fields. The symmetry operators $\boldsymbol{T}\left(\ell_{x}\right)$, $\boldsymbol{Z}, \boldsymbol{S}$ and $\boldsymbol{\Omega}$ are given in $(5.1 a),(5.1 b),(7.1 a)$ and $(7.1 b)$. Flowfield visualizations of $\mathrm{TVF}_{1}$ and $\mathrm{TVF}_{2}$ are given in figure 2, WVF, TWI and wTWI in figure 8, oWVF in figure 9 and SVF in figure 10. For a depiction of RIB, see Nagata (2013).

Twist (TWI) and wavy twist (wTWI) vortex flows emerge from the twist and wavy twist instabilities of $\mathrm{TVF}_{1}$ (Weisshaar et al. 1991; Antonijoan \& Sánchez 2000). Like WVF, wTWI has the symmetries $\boldsymbol{S}$ and $\boldsymbol{\Omega}$ but loses mirror symmetry $\boldsymbol{Z}$. TWI retains the mirror symmetry $\boldsymbol{Z}$ of $\mathrm{TVF}_{1}$ and has shift-rotation symmetry $\boldsymbol{\Omega}$, but not shift-reflection $S$. TWI therefore breaks only the continuous-symmetry $\boldsymbol{T}\left(\ell_{x}\right)$ of $\mathrm{TVF}_{1}$, while WVF and wTWI emerge in discrete-symmetry-breaking (DSB) pitchfork bifurcations from $\mathrm{TVF}_{1}$, breaking $\boldsymbol{Z}$. Both TWI and wTWI are comprised of counter-propagating streaks with arrangements of vortices embedded between the streaks, with the vortices of wTWI staggered whilst the vortices of TWI are not. The flowfields of a representative set of WVF, TWI and WTWI are depicted in figure 8.

The complex-conjugate eigenvalues of the oscillatory wavy instability instigate a Hopf bifurcation from $\mathrm{TVF}_{1}$ towards a periodic orbit of the Navier-Stokes equations. To our knowledge, calculations of the oscillatory flow bifurcating directly from $\mathrm{TVF}_{1}$ have not been previously reported in the literature for Taylor-Couette flow or RPCF though the oscillatory instability has been known since Nagata (1988). However, Hopf bifurcations from tertiary states have been reported, such as those discussed in Nagata \& Kawahara (2004), and we will make a distinction between those quaternary states and the time-periodic tertiary state. We herein refer to the latter as oscillatory wavy vortex flow (oWVF), since its flowfield resembles that of a steady wavy vortex which oscillates in time. Flowfield depictions of a stable oWVF state at two points during its oscillation are shown in figure 9. Its structure is much simpler than the periodic orbits found in non-rotating subcritical PCF by, for example, Kawahara \& Kida (2001) and Viswanath (2007), though its $E_{c f}$ evolution is reminiscent of the orbit denoted $\overline{1}$ in symbolic dynamics by Kreilos \& Eckhardt (2012). We find that oWVF holds the same symmetries as WVF and wTWI: shift-reflection $\boldsymbol{S}$ and shift-rotation $\boldsymbol{\Omega}$. However, the orbit does not completely break mirror symmetry $\boldsymbol{Z}$; in fact it preserves a version of $\boldsymbol{Z}$ as a space-time symmetry whereby the solution is invariant to advancing half a period and mirroring in $z$, as can be seen in the flowfields of figure 9. 

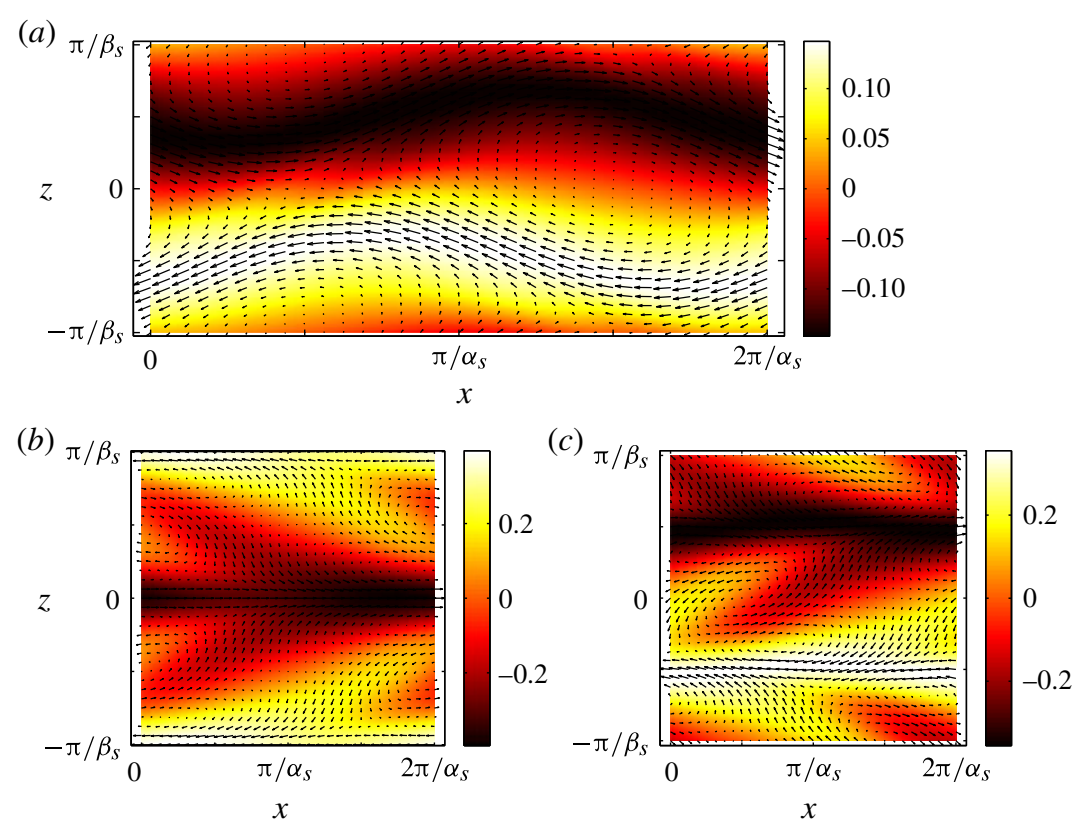

Figure 8. (Colour online) A representative set of flowfield images of the known tertiary states which bifurcate from $\mathrm{TVF}_{1}$ : (a) Wavy vortex flow (WVF) with $\left(\alpha_{s}, \beta_{s}, R e, R o\right)=$ $(0.7,1.5,100,0.05)$; $(b)$ twist vortex flow (TWI) with $\left(\alpha_{s}, \beta_{s}, R e, R o\right)=(1.5,1.5,100,0.7)$; (c) wavy twist vortex flow (wTWI) with $\left(\alpha_{s}, \beta_{s}, R e, R o\right)=(1.5,1.5,100,0.7)$. The $(x, z)$ cross-section at $y=0$ of each three-dimensional velocity field is depicted, and each flowfield has a true aspect ratio. Colours represent wall-normal velocity $v$, and the arrows comprise a vector plot of the streamwise $u$ and spanwise $w$ velocities.
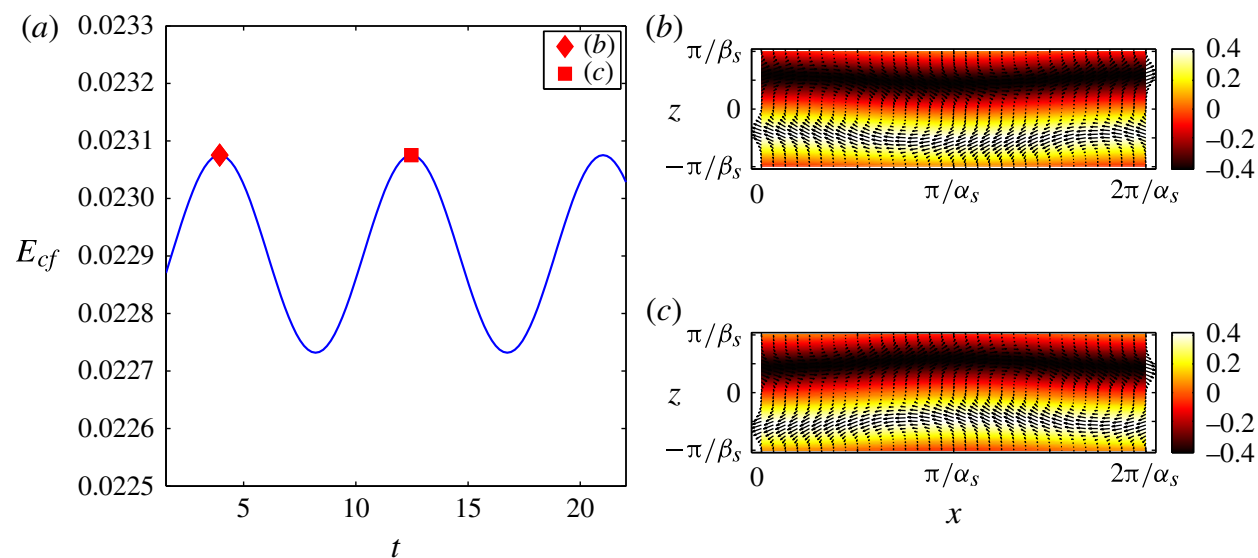

FIgURE 9. (Colour online) $E_{c f}$ energy evolution $(a)$ and flowfields $(b, c)$ of the oscillatory wavy vortex flow (oWVF) with period $T=17.09$ and parameters $\left(\alpha_{s}, \beta_{s}, \operatorname{Re}, \operatorname{Ro}\right)=$ $(0.9,3,100,0.55)$.

Skewed vortex flow is the steady streamwise-independent flow which bifurcates from $\mathrm{TVF}_{1}$ via the skew instability. SVF breaks mirror symmetry $\boldsymbol{Z}$, like the previous tertiary states excluding TWI, though its streamwise invariance allows it to satisfy 
(a)

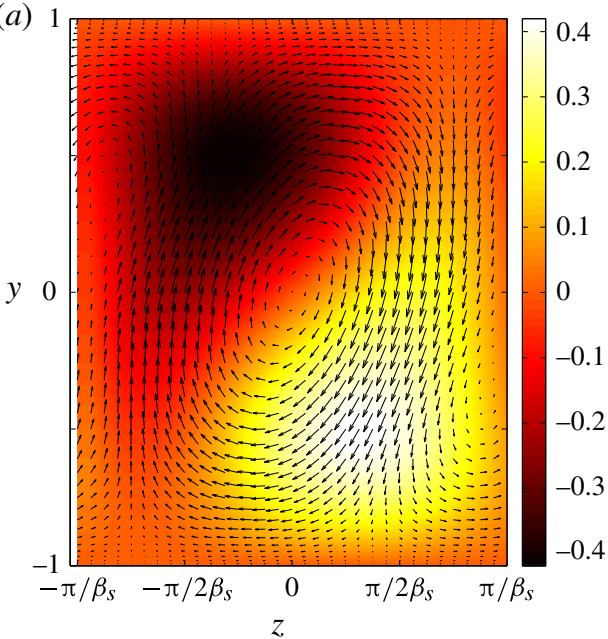

(b)

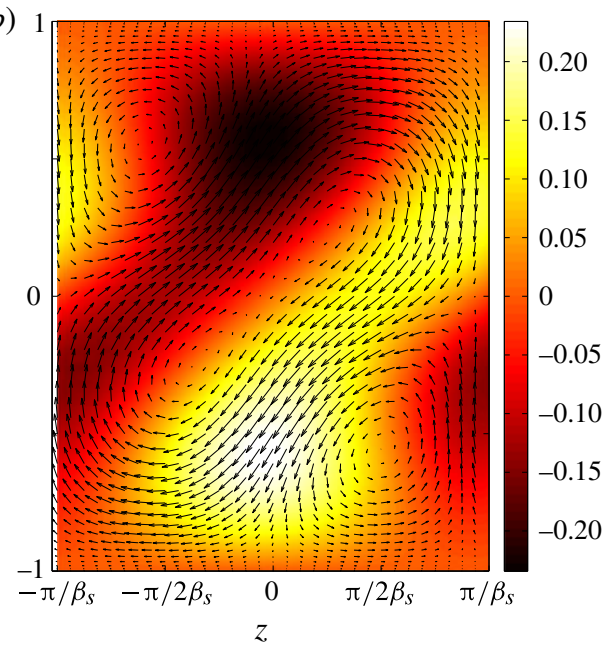

FIGURE 10. (Colour online) Skewed vortex flow (SVF) for $\left(\beta_{s}, R e\right)=(4,100)$ and (a) $R o=0.1$ and $(b) R o=0.2$. The flowfields are depicted with a colour plot of $u$ and a vector plot of $(v, w)$, in the $(y, z)$-plane. The flowfields consist of streamwise-independent counter-propagating streaks, with each streak skewed in the $(y, z)$-plane.

the continuous symmetry $\boldsymbol{T}\left(\ell_{x}\right)$. The spanwise skewness of the vortices is readily apparent in the velocity fields pictured in figure 10. We are unaware of SVF being reported by other authors.

\section{Bifurcation of oWVF}

We compute solution branches in $R o$ of oWVF in figure 11, for various $R e$ and $\left(\alpha_{s}, \beta_{s}\right)=(0.8,3)$ fixed. In the figure, we see the collision and merger of an unstable island of oWVF with a stable branch of oWVF solutions. The oWVF states appear for $R e \approx 92$ when $\mathrm{TVF}_{1}$ loses stability to a complex-conjugate eigenvalue pair, instigating a Hopf bifurcation towards oWVF. We herein refer to this set of solutions as the Hopf branch. Also for $R e=92$, a small island of oWVF solutions is present in $\left(R o, E_{c f}\right)$, with no solution set connecting the island to the Hopf branch at this fixed Reynolds number. Though there is no connection in $\left(R o, E_{c f}\right)$ between the island states and the oWVF branch which bifurcates from $\mathrm{TVF}_{1}$, the island states do themselves originate in bifurcations from $\mathrm{TVF}_{1}$. At fixed $R o$, a smooth bifurcation curve can be traced in $\left(R e, E_{c f}\right)$ which connects the island states to Hopf branch oWVF states which bifurcate directly from $\mathrm{TVF}_{1}$. As $R e$ increases from $R e=92$, the island of oWVF solutions grows over $\left(R o, E_{c f}\right)$, until for $R e=100$ the island and the Hopf branch collide.

In figure 12, we fix the wavenumber $\beta_{s}=3$ and Reynolds number $R e=100$ to explore bifurcations of oWVF in $\left(\alpha_{s}, R o\right)$. Here we find an analogous process to that of figure 11, with an island of unstable solutions emerging alongside the attractor states which bifurcate from $\mathrm{TVF}_{1}$. From figure 12, the collision between the island and the stable branch can be seen to occur for $\alpha_{s} \approx 0.8$. Thus, the bifurcation scenario is found from bifurcations in $\left(\alpha_{s}, R o\right)$ and $(R e, R o)$, suggesting that the process of collision and merger of stable and unstable oWVF solution sets is a codimension-two bifurcation.

Prior to the collision of the island and Hopf branch, stability calculations show that the Hopf branch is stable to harmonic perturbations, i.e. perturbations with $\alpha=\beta=0$ 


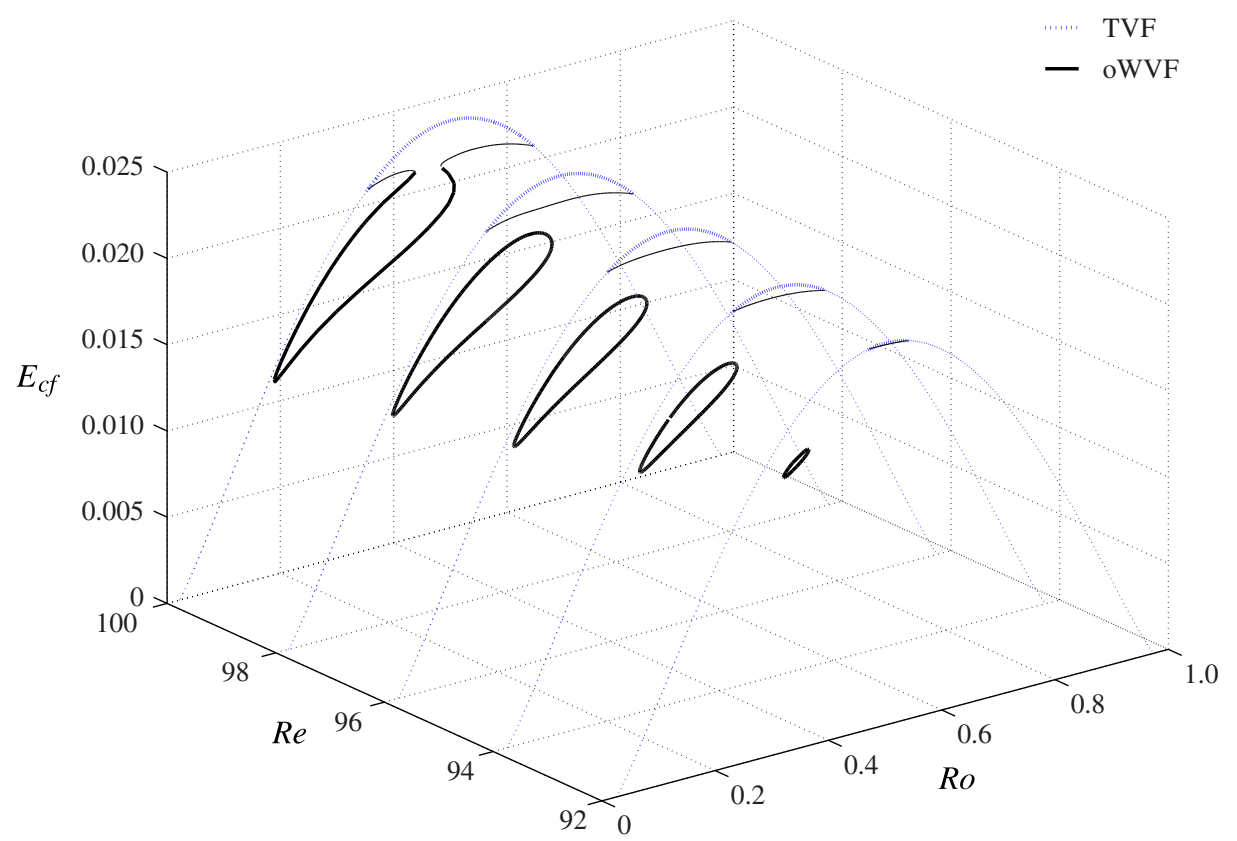

FIgURE 11. (Colour online) oWVF bifurcations in $R e$ and $R o$ for $\left(\alpha_{s}, \beta_{s}\right)=(0.8,3)$. The mean $E_{c f}$ over one period is used to denote the oWVF periodic orbits. Stable/unstable solutions are represented by thin/thick lines. An unstable island of oWVF solutions emerges for $R e \approx 92$ and collides with the stable oWVF branch for $R e \approx 100$ to create a region where a stable state no longer exists.

in (4.12). However, the oWVF states on the island are unstable. In figure 13 we plot the unstable growth rates for each solution on the $R e=98$ island. All solutions on the island are unstable, with a symmetry-breaking mode, which breaks both $S$ and $\boldsymbol{\Omega}$, destabilizing each state. A second instability mode, which preserves the $\boldsymbol{S}, \boldsymbol{\Omega}$ symmetries, destabilizes the upper branch of the island. Time marching of the NavierStokes equations constrained to the $\boldsymbol{S}, \boldsymbol{\Omega}$ symmetry subspace shows that the upperbranch symmetry-preserving instability initiates a bifurcation towards the stable lowerbranch island solution. This reveals that the island is in fact two connecting saddlenodes, with the upper branch a saddle and the lower branch a node. Time marching without the symmetry restrictions causes a bifurcation to either $\mathrm{TVF}_{1}$ or the Hopf branch oWVF states, depending on which are stable at the given rotation number.

In figure 14 we plot the unstable modes of the $R e=100$ oWVF solution branch, with the merged Hopf branch and island. The unstable island upper branch has now broken in two, so that the continuous solution branch now consists of two Hopf branches, two saddle branches and a node branch. The node branch contains a region of symmetry-preserving instability near the low Ro saddle-node point, and symmetry-constrained time marching of the Navier-Stokes equations shows that the mode initiates a period-doubling bifurcation. We do not further investigate the symmetry-preserving bifurcations here, rather we focus on the effect of the symmetry-breaking mode. Figure 14 shows that the symmetry-breaking mode destabilizes a large portion of the 'lower branch' of the merged oWVF states, and indeed there is a region $0.4382<R o<0.4834$ where no stable oWVF state 


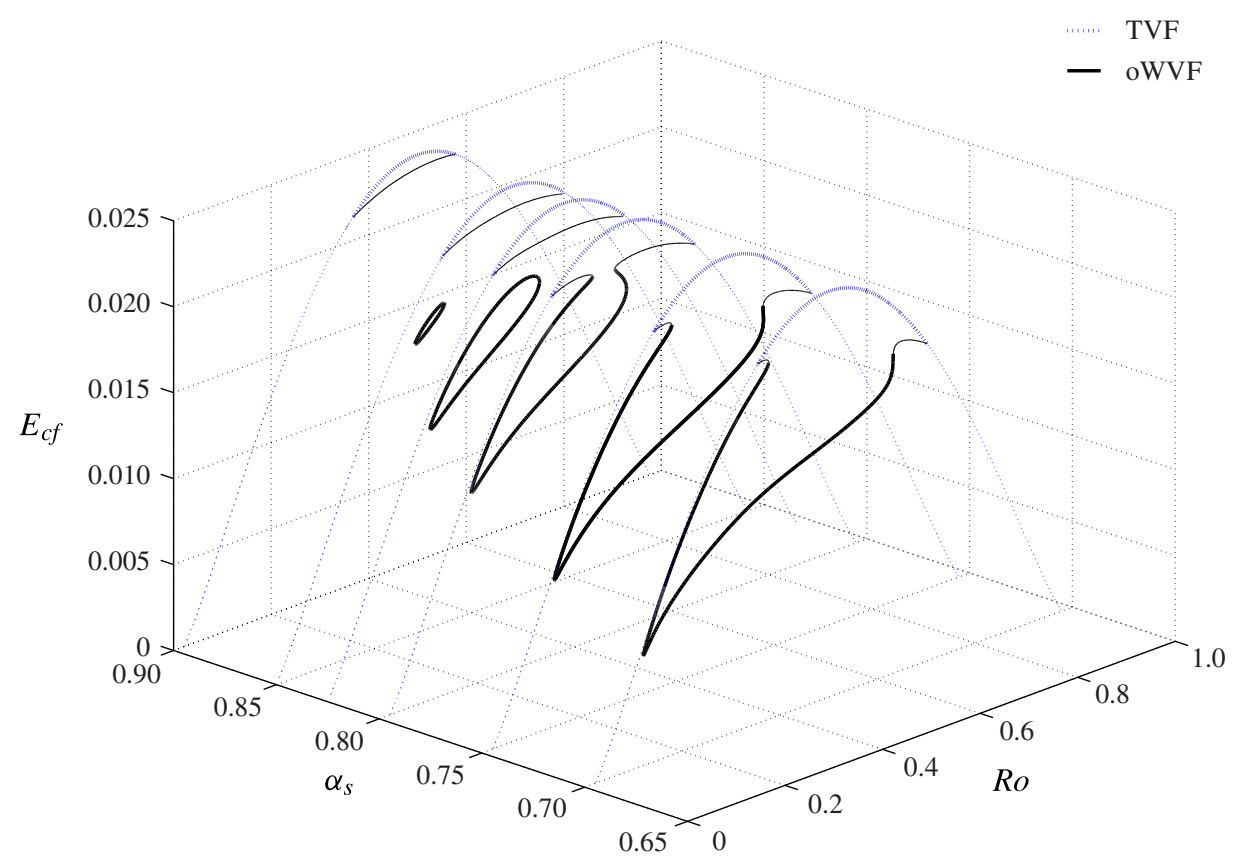

FIgURE 12. (Colour online) oWVF bifurcations in $\alpha_{s}$ and $R o$ for $\left(\beta_{s}, R e\right)=(3,100)$. The mean $E_{c f}$ over one period is used to denote the oWVF periodic orbits. Stable/unstable solutions are represented by thin/thick lines. An unstable island of oWVF solutions emerges for $\alpha_{s} \approx 0.829$ and collides with the stable oWVF branch for $\alpha_{s} \approx 0.8$ to create a region where a stable state no longer exists.

exists. Moreover, $\mathrm{TVF}_{1}$ is also unstable in this region, meaning no stable state which bifurcates via $\mathrm{TVF}_{1}$ remains. Therefore, an important consequence of the collision between the island and the Hopf branch is to create a parameter region where no stable solution exists.

To show the effect of the symmetry-breaking instability on the lower-branch unstable oWVF states for $R e=100$, we use the total kinetic energy density $E$, the bulk viscous dissipation rate $D$ and the wall-shear power input $I$ :

$$
\begin{gathered}
E=\frac{1}{2 V} \int_{V}|\boldsymbol{u}+\boldsymbol{U}|^{2} \mathrm{~d} \boldsymbol{x}, \\
D=\frac{1}{2 V} \int_{V}|\boldsymbol{\nabla} \times(\boldsymbol{u}+\boldsymbol{U})|^{2} \mathrm{~d} \boldsymbol{x}, \\
I=1+\frac{1}{2 A} \int_{A}\left(\left.\frac{\partial u}{\partial y}\right|_{y=1}+\left.\frac{\partial u}{\partial y}\right|_{y=-1}\right) \mathrm{d} x \mathrm{~d} z,
\end{gathered}
$$

to trace trajectories away from lower-branch unstable oWVF states at various $R o$ in the $(I, D)$-plane. The total energy time derivative can be written in terms of $I$ and $D$ :

$$
\frac{\mathrm{d} E}{\mathrm{~d} t}=I-D .
$$

Any equilibrium state must therefore lie on the line $I=D$, and any periodic orbit must form a closed loop in the (I,D)-plane (Gibson et al. 2008). In figure 15( $a-c)$, 


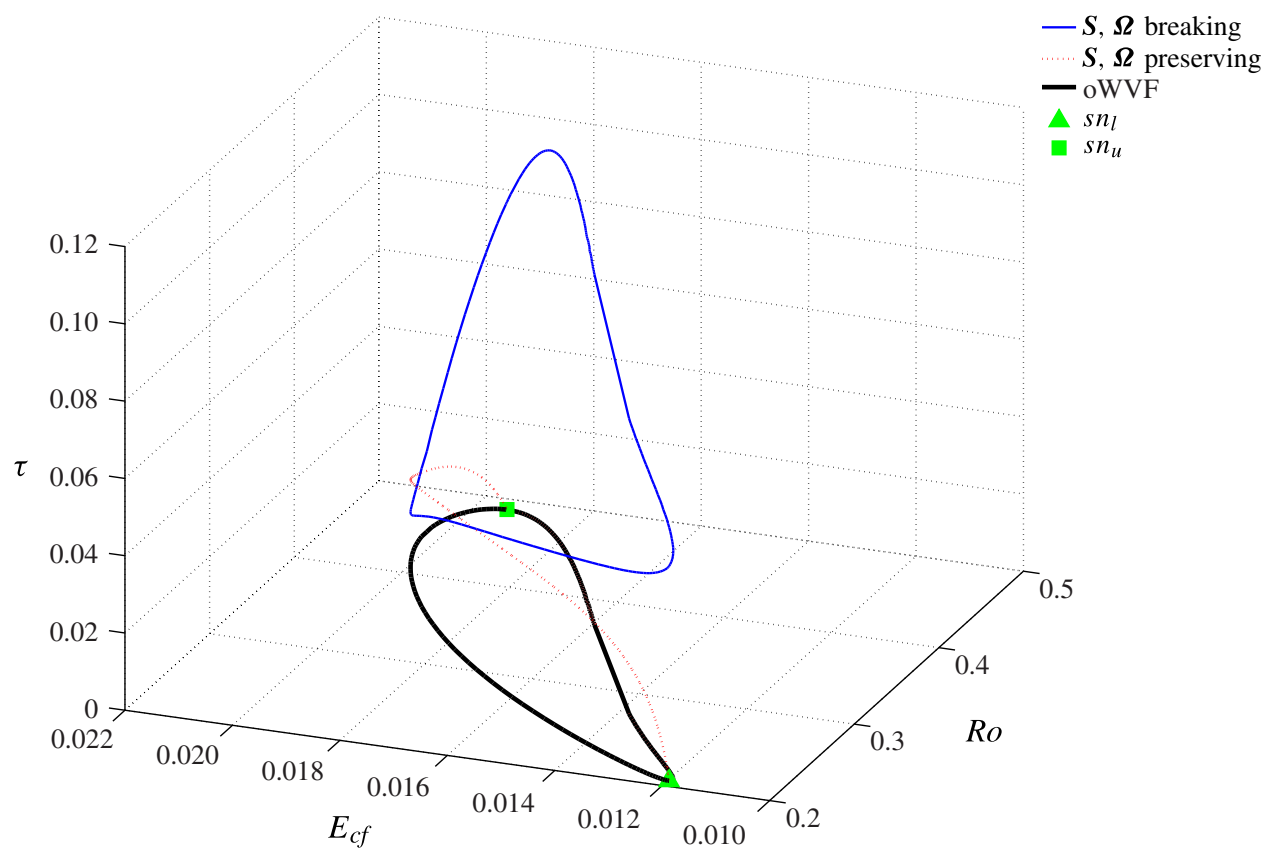

FIgURE 13. (Colour online) Stability of the oWVF island for $\left(\alpha_{s}, \beta_{s}, R e\right)=(0.8,3,98)$ from figure 11. The $\left(R o, E_{c f}\right)$ bifurcation of oWVF is plotted in the plane $\tau=0$, with the lower and upper $R o$ saddle-node points, $s n_{l}$ and $s n_{u}$, denoted by a triangle and square respectively. For each $\left(R o, E_{c f}\right)$ coordinate on the island, we plot the unstable harmonic growth rates $\tau>0$ of the oWVF solutions, i.e. for detuning parameters $\alpha=\beta=0$ in (4.12). Each instability mode is stationary, with zero imaginary part. The solid line (blue) corresponds to a symmetry-breaking mode, whilst the dotted (red) mode preserves the $\boldsymbol{S}, \boldsymbol{\Omega}$ symmetries of the oWVF solutions. The island can be divided into an upper and lower branch, with the upper/lower branch unstable/stable to a symmetry-preserving mode. Both branches are, however, destabilized by the symmetry-breaking mode.

we plot unstable mode trajectories in the $(I, D)$-plane for lower-branch oWVF states at $R o=0.25,0.45,0.5$, respectively. These rotation numbers are chosen since each corresponds to a different case: for $R o=0.25 \mathrm{TVF}_{1}$ is stable; $R o=0.45$ lies in the region without any stable state; and for $R o=0.5$ there is a stable oWVF state. For $R o=0.25$ there are two oWVF states, which are labelled $\mathrm{oWVF}_{L B}$ and $\mathrm{oWVF}_{U B}$ in figure $15(a)$, according to their cross-flow energies. We carry this notation into figure $15(b)$, where only $\mathrm{oWVF}_{L B}$ remains, and figure $15(c)$ where the upper- and lower-branch solutions are joined by a third, stable oWVF state. Each projection in the figure is generated from a time series of the unstable oWVF $\mathrm{WB}_{L B}$ state evolved forwards in time until it destabilizes. In cases $(a)$ and $(c)$, the trajectories move towards the stable $\mathrm{TVF}_{1}$ and oWVF states respectively. The trajectory in figure $15(b)$, however, points to the existence of a homoclinic orbit (Wiggins 2003) or homoclinic tangle about $\mathrm{oWVF}_{L B}$, as the symmetry-breaking instability causes the oWVF state to break down, but with no stable state in the system the flow eventually returns to oWVF $\mathrm{F}_{L B}$, and the cycle repeats again.

In figure 16 we show that the stable $\mathrm{TVF}_{1}$, the trajectory around $\mathrm{oWVF}_{L B}$ and the stable oWVF of figure $15(a-c)$ are attractors of small perturbations of the flow. Figure $16(a-c)$ shows the $E_{c f}$ evolution of an initial condition comprised of 


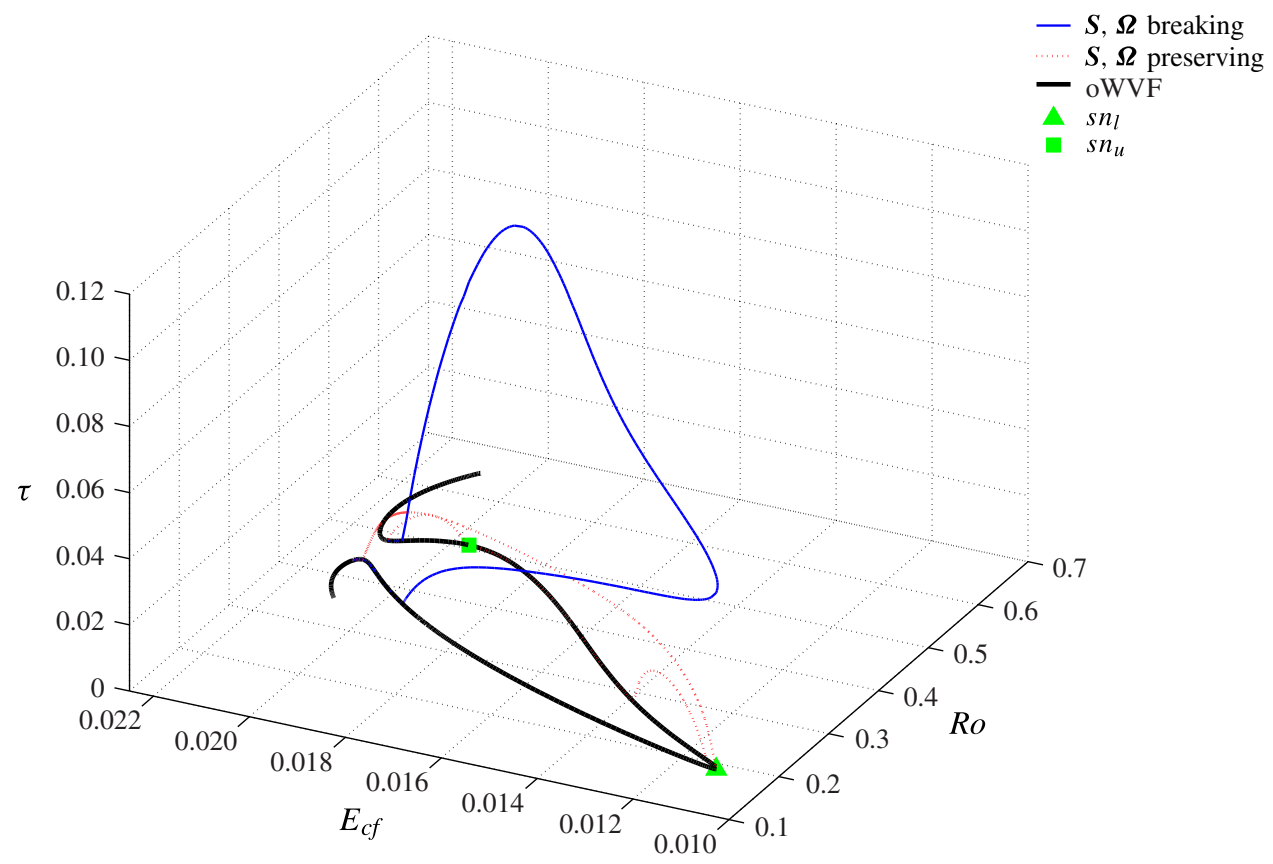

FIGURE 14. (Colour online) Stability of the oWVF bifurcation curve for $\left(\alpha_{s}, \beta_{s}, R e\right)=$ $(0.8,3,100)$ of figures 11 and 12 . We plot the unstable harmonic modes, as in figure 13 , with the $\left(R o, E_{c f}\right)$ bifurcation of oWVF plotted in the plane $\tau=0$. The lower and upper $R o$ saddle-node points, $s n_{l}$ and $s n_{u}$, are denoted by a triangle and square respectively. The tertiary growth rate $\tau>0$ is then plotted at each point of the $\left(R o, E_{c f}\right)$-coordinates of the oWVF solutions. Each instability mode is stationary, with zero imaginary part. The solid line (blue) curve corresponds to a symmetry-breaking mode, whilst the dotted (red) mode preserves the $\boldsymbol{S}, \boldsymbol{\Omega}$ symmetries of the oWVF solutions.
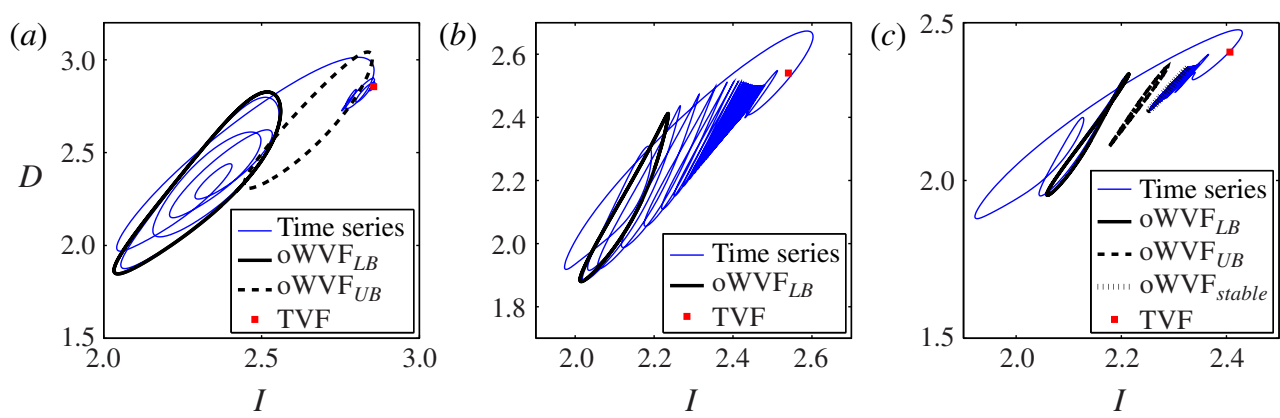

FIGURE 15. (Colour online) $(I, D)$-projections of time evolution away from unstable lower-branch oWVF states for $\left(\alpha_{s}, \beta_{s}, R e\right)=(0.8,3,100)$. (a) $R o=0.25$. In this case $\mathrm{TVF}_{1}$ is the only attractor in the system, and the trajectory away from $\mathrm{oWVF}_{L B}$ avoids $\mathrm{oWVF}_{U B}$ and moves towards this stable state. $(b) R o=0.45$. At this rotation number there are no stable states, only unstable $\mathrm{TVF}_{1}$ and $\mathrm{oWVF}_{L B}$. The trajectory away from oWVF $\mathrm{WB}_{L}$ appears to bypass $\mathrm{TVF}_{1}$ and return to $\mathrm{oWVF}_{L B}$. (c) $R o=0.5$. Here there is a stable oWVF state, which is found by the trajectory away from oWVF $\mathrm{WB}_{L B}$. 

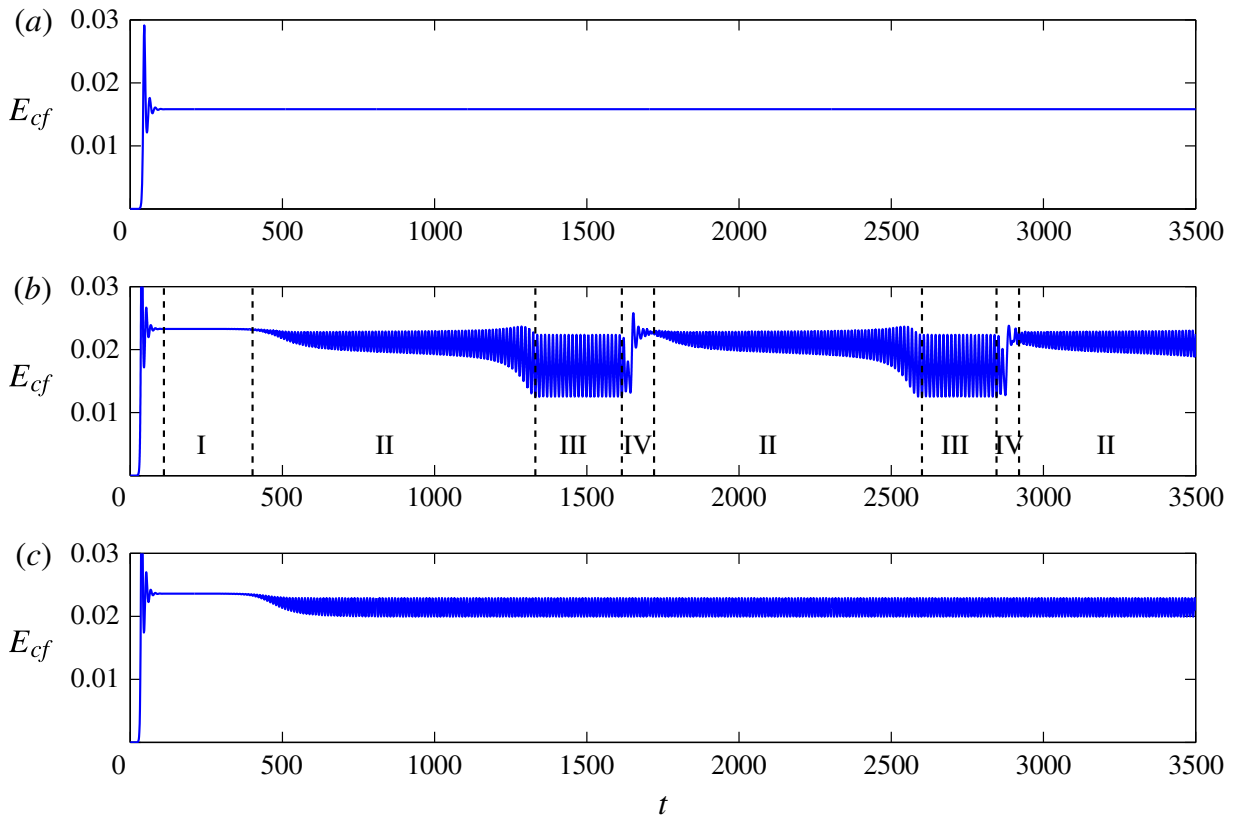

FIGURE 16. (Colour online) $E_{c f}$ evolution of a random initial condition for the parameter cases of figure 15. (a) $R o=0.25$ : smooth approach to stable $\mathrm{TVF}_{1}$. (b) $R o=0.45$ : approach to a homoclinic orbit about $\mathrm{oWVF}_{L B}$. No stable state exists at these parameters, with LAM, $\mathrm{TVF}_{1}$ and $\mathrm{oWVF}_{L B}$ all unstable. There are three phases of the cycle: (I) $\mathrm{TVF}_{1}$, (II) a trajectory on the unstable manifold of $\mathrm{TVF}_{1}$, (III) $\mathrm{oWVF}_{L B}$ and (IV) a transition region away from oWVF. The flow moves from (I) to (II) and then cycles from (III) to (IV) and (II). (c) $R o=0.5$ : smooth approach to stable oWVF via $\mathrm{TVF}_{1}$.

Stokes modes, i.e. divergence-free solutions of the Stokes operator. It is particularly interesting that the trajectory of figure $15(b)$ is an attractor as shown in $16(b)$. It is clear from the figure that the unstable manifold of $\mathrm{TVF}_{1}$ connects with the stable manifold of $\mathrm{oWVF}_{L B}$ indicating a heteroclinic connection from $\mathrm{TVF}_{1} \rightarrow \mathrm{oWVF}_{L B}$. This is denoted (I) $\rightarrow$ (II) $\rightarrow$ (III) in figure $16(b)$. The unstable manifold of $\mathrm{oWVF}_{L B}$ appears to then connect directly to the unstable manifold of $\mathrm{TVF}_{1}$, denoted $(\mathrm{IV}) \rightarrow$ (II) in the figure, which suggests there may be a heteroclinic connection from $\mathrm{oWVF}_{L B} \rightarrow \mathrm{TVF}_{1}$. We have tried exhaustively to find such a heteroclinic connection from $\mathrm{oWVF}_{L B}$ to $\mathrm{TVF}_{1}$; however, in none of our simulations were we able to find a trajectory which closely approached the unstable $\mathrm{TVF}_{1}$.

Were there to be such a heteroclinic connection, it would have implied that there was a homoclinic orbit about the equilibrium $\mathrm{TVF}_{1}$, not just the periodic orbit $\mathrm{oWVF}_{L B}$, which could then have been directly susceptible to the Shilnikov phenomenon (Shilnikov 1965). The lack of a heteroclinic orbit might then imply that $\mathrm{oWVF}_{L B}$ and $\mathrm{TVF}_{1}$ are connected in a heteroclinic tangle. The creation of the attractor in figure $16(b)$ can then be understood through a scenario similar to the supercritical bifurcation cascade of travelling waves in pipe flow by Mellibovsky \& Eckhardt (2012), where a chaotic attractor is created and destroyed by heteroclinic tangencies rather than heteroclinic orbits. We therefore suggest that the collision of the Hopf branch and island of oWVF states creates heteroclinic tangencies between oWVF and $\mathrm{TVF}_{1}$ states, allowing a mildly chaotic attractor to occur. The emergence 

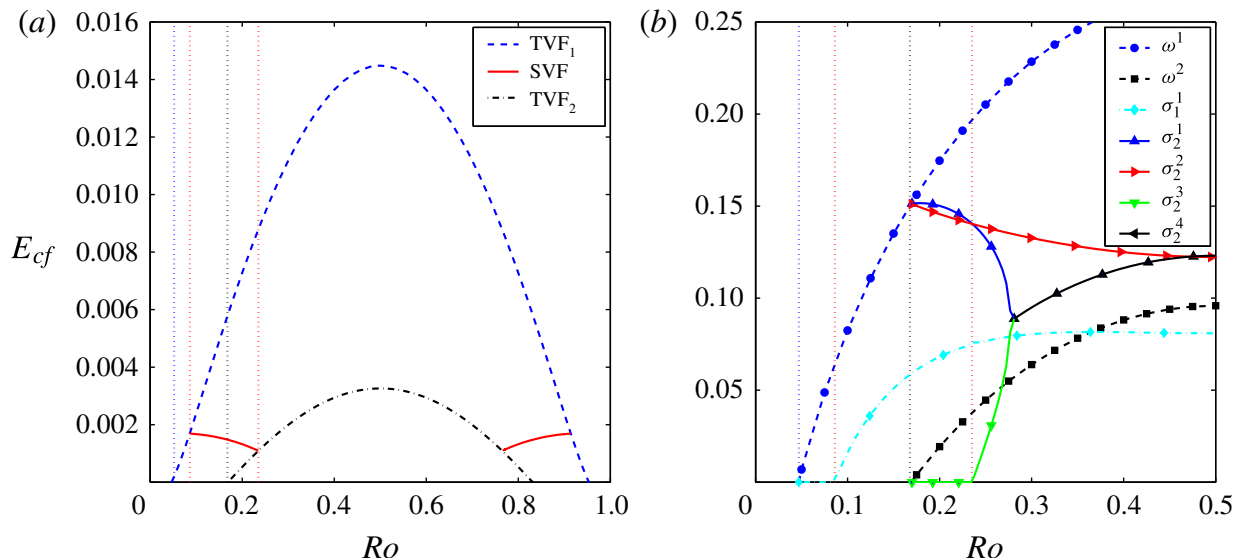

FIGURE 17. (Colour online) SVF bifurcations for $\left(\alpha_{s}, \beta_{s}, R e\right)=(0,4,100)$. (a) Bifurcation of SVF from $\mathrm{TVF}_{1}$ to $\mathrm{TVF}_{2}$. (b) Real parts of the linear instability modes of LAM, TVF and $\mathrm{TVF}_{2} . \omega^{i}$ denotes the $i$ th unstable mode of LAM, while $\sigma_{j}^{i}$ denotes the $i$ th unstable mode of the $j$ th TVF solution. The stability operators of each state also contain stable modes, which would have negative real part; however, these modes are not included in the figure. Here $\omega^{1}, \omega^{2}, \sigma_{1}^{1}, \sigma_{2}^{1}, \sigma_{2}^{2}, \sigma_{2}^{3}$ are stationary modes while $\sigma_{2}^{4}$ is an oscillatory mode. The four vertical dashed lines indicate, from the left: the emergence of $\mathrm{TVF}_{1}$, the emergence of SVF, the emergence of $\mathrm{TVF}_{2}$ and the loss of SVF. Comparing $(a)$ and $(b)$, we see that SVF emerges as $\sigma_{1}^{1}$ crosses the zero axis and disappears as $\sigma_{2}^{3}$ crosses the zero axis.

of this attractor points to an interesting route to chaos in this parameter region of RPCF, potentially distinct from the known routes in non-dissipative systems, such as the breakdown of two-tori and period-doubling cascades in Taylor-Couette flow (Abshagen et al. 2005; Lopez \& Marques 2005).

\section{Bifurcation of SVF}

In figure $17(a)$ we plot bifurcations of streamwise-independent structures which bifurcate from $\mathrm{TVF}_{1}$ with $\beta_{s}=4$. We find that $\mathrm{SVF}$ exists for a short range of high and low $R o$. At $R o=0.085, \mathrm{SVF}$ bifurcates from $\mathrm{TVF}_{1}$ in a DSB pitchfork bifurcation; however, at $R o=0.235$, SVF bifurcates from the second Taylor vortex solution, $\mathrm{TVF}_{2}$, also in a DSB pitchfork bifurcation. The stability of LAM and harmonic stability of both $\mathrm{TVF}_{1}$ and $\mathrm{TVF}_{2}$ with $\beta_{s}=4$ are indicated in figure $17(b)$, where we use the notation $\omega^{i}$ to denote the $i$ th unstable mode of $\mathrm{LAM}$, and $\sigma_{j}^{i}, i, j \in \mathbb{N}$, to denote the $i$ th unstable mode of the $j$ th TVF solution.

We find that $\mathrm{TVF}_{1}$ has only one unstable mode, $\sigma_{1}^{1}$, which has zero phase $\left(\sigma_{i}=0\right)$, and SVF bifurcates from $\mathrm{TVF}_{1}$ when stability is lost to $\sigma_{1}^{1} \cdot \mathrm{TVF}_{2}$ has four unstable modes: $\sigma_{2}^{1}$ and $\sigma_{2}^{2}$ destabilize $\mathrm{TVF}_{2}$ as it bifurcates from LAM, $\sigma_{2}^{3}$ appears when Ro is increased, then $\sigma_{2}^{1}$ and $\sigma_{2}^{3}$ merge to form a pair of complex modes which we label $\sigma_{2}^{4}$. It appears that $\sigma_{2}^{1}$ and $\sigma_{2}^{2}$ are inherited from LAM, since $\omega^{1}=\sigma_{2}^{1}=\sigma_{2}^{2}$ at the point where $\mathrm{TVF}_{2}$ first bifurcates from LAM. We find that $\mathrm{SVF}$ bifurcates from $\mathrm{TVF}_{2}$ when $\sigma_{2}^{3}$ appears, implying that SVF connects the two Taylor vortex solutions. Furthermore, the SVF flowfield more closely resembles $\mathrm{TVF}_{2}$ than $\mathrm{TVF}_{1}$ as $R o$ approaches 0.235 . The SVF flowfield in figure $10(a)$, with $R o=0.1$, has one row of vortices in the wallnormal direction, whereas in figure $10(b)$, with $R o=0.2$, a double layer of vortices 

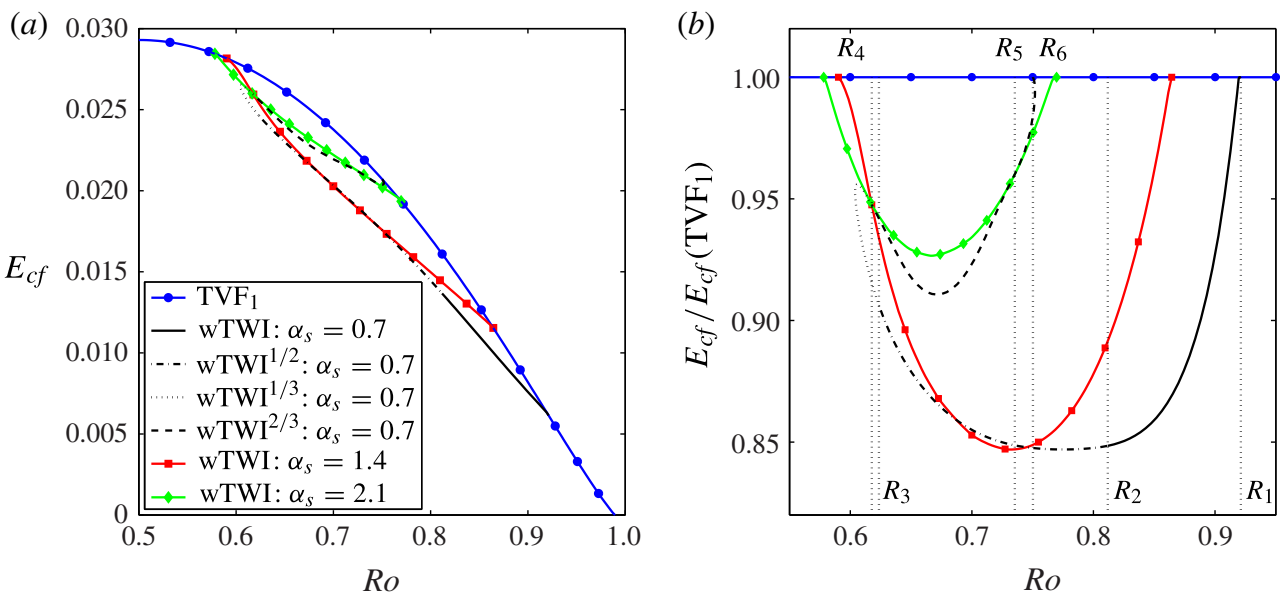

FIGURE 18. (Colour online) wTWI bifurcations under rotation with $\beta_{s}=1.5, R e=$ 100. (a) Bifurcations under Ro with $E_{c f}$ as solution measure. (b) Tertiary bifurcations normalized by the cross-flow energy of $\mathrm{TVF}_{1}$. All solutions undergo pitchfork bifurcations from $\mathrm{TVF}_{1}$; however, the $\alpha_{s}=0.7$ makes an excursion along defected wTWI states before re-joining with $\mathrm{TVF}_{1}$. The defected states are represented with the notation $\mathrm{wTWI}^{i / j}, i, j \in$ $\mathbb{N}$, to denote a wavy twist arrangement of $j$ vortices per streamwise wavelength, with $i$ 'strong' vortices in that arrangement. Visualizations of the defected states are given in figure 19. The rotation numbers $R_{1}, R_{2}, \ldots, R_{6}$ labelled in $(b)$ demarcate the bifurcation points along the $\alpha_{s}=0.7$ continuation curve, where the states change behaviour.

emerges in similarity with $\mathrm{TVF}_{2}$ flowfields. The SVF solution occurs again for high $R o$, close to the point when linear stability of the base flow is regained for strong rotation. In this high-Ro region, $\mathrm{SVF}$ again connects the $\mathrm{TVF}_{1}$ and $\mathrm{TVF}_{2}$ states.

\section{Defects and localization of wavy twist vortex flow}

We now turn our attention to bifurcations of wTWI, and show that under bifurcations in Ro, a set of defected wTWI states arises. We characterize defected solutions in terms of the streamwise arrangement of streamwise-spanwise oriented vortices and their differing strengths. A strict definition of the defects characterized in this paper is given in the following discussion. In figure 18 we show how wTWI with $\beta_{s}=1.5$ bifurcates from $\mathrm{TVF}_{1}$ at $\alpha_{s}=0.7,1.4,2.1$. Figure 18(a) shows the bifurcations under $R o$ with $E_{c f}$ as solution measure, while in figure $18(b)$ we have normalized the branches with respect to $\mathrm{TVF}_{1}$ for clarity. For each $\alpha_{s}$, the wTWI solution branches are discrete-symmetry-breaking pitchforks from $\mathrm{TVF}_{1}$. However, we find that along the $\alpha_{s}=0.7$ solution branch the wTWI solutions undergo a series of phenomenological transformations, with the flowfield changing its appearance a number of times. To characterize each solution, we introduce the notation $\mathrm{WTWI}^{i / j}, i, j \in \mathbb{N}$, to denote a wavy twist arrangement of $j$ vortices per streamwise wavelength, with $i$ 'strong' vortices in that arrangement. We define a defected wTWI to be any state with $i<j$, such that $\mathrm{wTWI}^{j / j}$ represents the classic wTWI solution over streamwise wavenumber $\alpha_{s} / j$. Using this classification, we encounter the defected states $\mathrm{WTWI}^{1 / 2}, \mathrm{WTWI}^{1 / 3}$ and $\mathrm{WTWI}^{2 / 3}$, and a visual representation of each is given in figure 19. Despite the changes in flowfield structure, the symmetries $\boldsymbol{S}$ and $\boldsymbol{\Omega}$ are satisfied by all the defected solutions. 

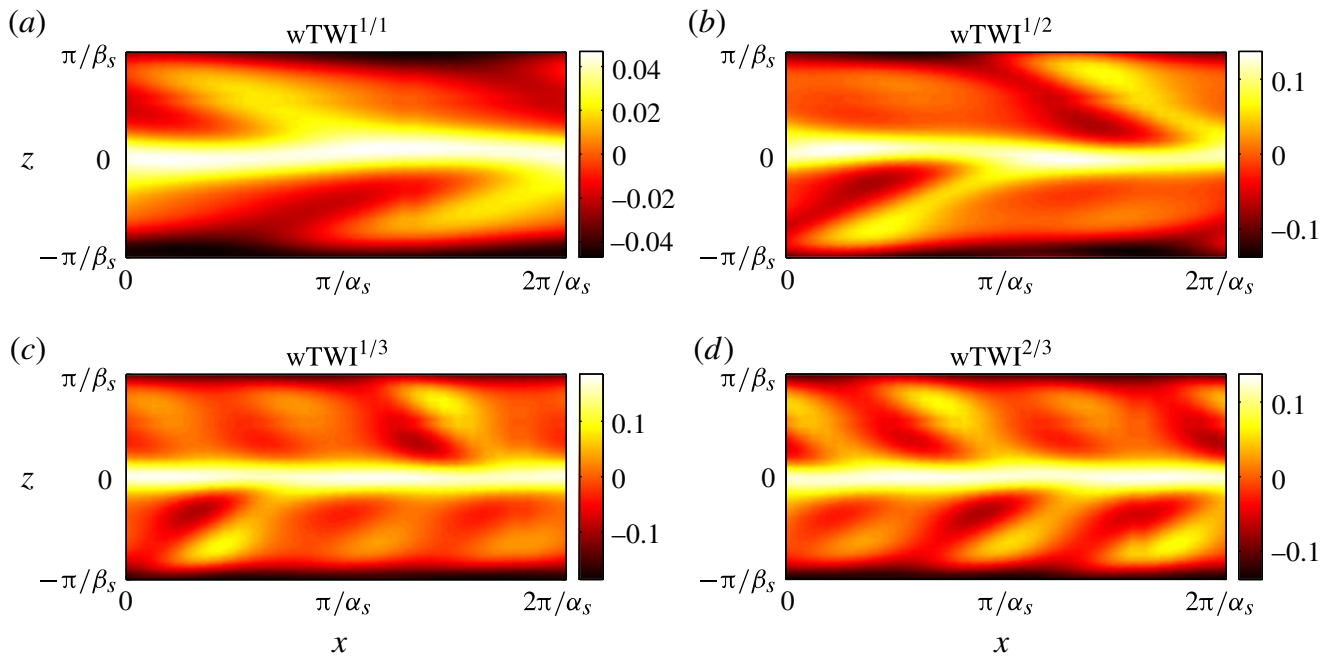

FIGURE 19. (Colour online) Flowfield visualizations of the defected wTWI solutions of the bifurcation in figure 18 , for $\left(\alpha_{s}, \beta_{s}, R e\right)=(0.7,1.5,100)$. Flowfields are depicted in the $(x, z)$-plane at $y=0$, with colour plots of $u$.

The classification of the defected WTWI in figure 18 can be made rather arbitrarily by inspection of the flowfield, but the precise point in $R o$ at which a state switches from one to another can be interpreted as a bifurcation point from Floquet analysis, with detuning streamwise wavenumber $\alpha=0.7$, of the secondary state $\operatorname{TVF}_{1}\left(\beta_{s}=1.5\right)$ and the tertiary states $\operatorname{wTWI}\left(\alpha_{s}=1.4\right) \equiv \mathrm{WTWI}^{2 / 2}\left(\alpha_{s}=0.7\right)$ and $\operatorname{WTWI}\left(\alpha_{s}=2.1\right) \equiv \mathrm{WTWI}^{3 / 3}\left(\alpha_{s}=0.7\right)$. In figure $18(b)$ the bifurcation points $R_{1}, R_{2}, \ldots, R_{6}$ are defined to indicate six rotation numbers from the bifurcation of WTWI $\left(\alpha_{s}=0.7\right)$ from $\mathrm{TVF}_{1}$ at $R_{1}=0.9192$ to the bifurcation of $\mathrm{WTWI}\left(\alpha_{s}=0.7\right)$ from $\mathrm{TVF}_{1}$ at $R_{6}=0.7502$. Each rotation number $R_{1}, R_{2}, \ldots, R_{6}$ can then be identified with a stability mode crossing the real axis in figure $20(a)$. It is straightforward that the pitchfork bifurcations of wTWI from $\mathrm{TVF}_{1}$ correspond to a stationary stability mode (solid line in figure $20 a$ ) crossing from negative to positive real part. Curiously, however, the changes in solution from wTWI ${ }^{1 / 1} \rightarrow \mathrm{wTWI}^{1 / 2}$ and $\mathrm{WTWI}^{1 / 2} \rightarrow \mathrm{wTWI}^{1 / 3}$ coincide with the subharmonic stability mode of $\mathrm{WTWI}^{2 / 2}$ crossing the zero real axis at $R_{2}$ and $R_{3}$, shown in dashed line in figure 20(a). This suggests that bifurcations occur at $R_{2}$ and $R_{3}$ which create the defected wTWI states. Floquet analysis of $\mathrm{wTWI}^{3 / 3}$ with $\alpha=0.7$ yields three instability modes, inherited from secondary instabilities of $\mathrm{TVF}_{1}$. The points at which the first and third of these modes cross from negative to positive real part correspond to the bifurcations of $\mathrm{WTWI}^{2 / 3}$ from wTWI $^{3 / 3}$ at $R_{4}$ and $R_{5}$, though we do not find any change in the solution flowfield between $R_{4}<R o<R_{6}$ where the second mode crosses the real axis.

Further insight into how the solution changes as it travels along the bifurcation curve can be obtained through analysis of the first three streamwise harmonic energies $E^{1}, E^{2}$ and $E^{3}$. To define these quantities, we take a solution with nonlinear perturbation velocity $\boldsymbol{u}_{s}$, and write its Fourier transform as

$$
v_{\alpha_{s} \beta_{s}}^{m n}=\int_{0}^{2 \pi / \alpha_{s}} \int_{0}^{2 \pi / \beta_{s}} \boldsymbol{u}_{s}(x, y, z) \exp \left(\mathrm{i}\left(m \alpha_{s} x+n \beta_{s} z\right)\right) \mathrm{d} z \mathrm{~d} x
$$



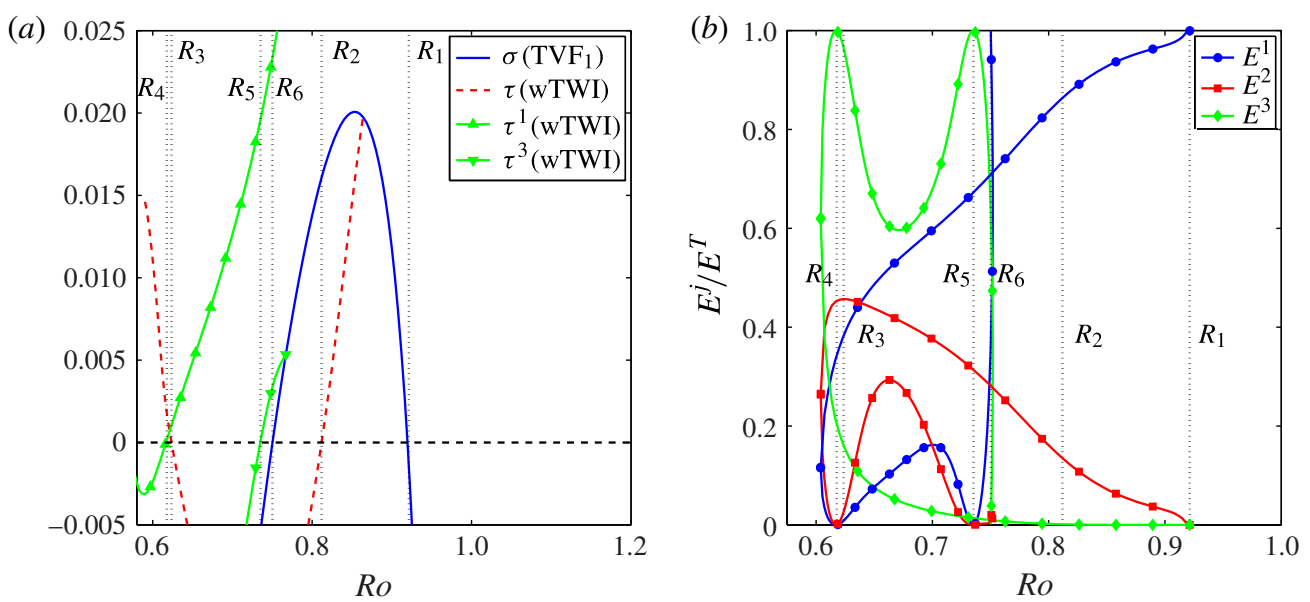

FIgURE 20. (Colour online) (a) Secondary and tertiary instability growth rates. Line (blue): secondary growth rate $\sigma$ of $\mathrm{TVF}_{1}$. Dashed line (red): tertiary growth rate $\tau$ of WTWI $\left(\alpha_{s}=1.4\right)$. Triangles (green): tertiary growth rates of the first (solid) and third (dashed) modes $\tau^{1}$ and $\tau^{3}$ of $\operatorname{WTWI}\left(\alpha_{s}=2.1\right)$. The dashed curve can be seen to connect with the solid line, indicating that the tertiary instability of $\operatorname{wTWI}\left(\alpha_{s}=1.4\right)$ is inherited from instability of $\mathrm{TVF}_{1}$. Further calculations (not shown) indicate similarly that $\tau^{1}$ and $\tau^{3}$ are inherited from instabilities of $\mathrm{TVF}_{1}$. (b) Streamwise harmonic energies expressed as ratios of $E^{T}=E^{1}+E^{2}+E^{3}$. The bifurcation points in each figure $R_{1}, R_{2}, \ldots, R_{6}$ correspond to a change in behaviour of $\mathrm{WTWI}\left(\alpha_{s}=0.7\right)$.

From this we calculate the spectral energy in a given Fourier mode as

$$
E_{\alpha_{s} \beta_{s}}^{m n}=\frac{1}{2} \int_{-1}^{1} \boldsymbol{v}_{\alpha_{s} \beta_{s}}^{m n}\left(\boldsymbol{v}_{\alpha_{s} \beta_{s}}^{m n}\right)^{*} \mathrm{~d} y
$$

where ${ }^{*}$ denotes the complex conjugate. We then define the $j$ th streamwise harmonic energy to be the sum over the spanwise spectral energies of the jth streamwise Fourier mode

$$
E^{j}=\sum_{n=-T_{n}}^{T_{n}} E_{\alpha_{s} \beta_{s}}^{j n},
$$

where $T_{n}$ is the numerical truncation in $z \cdot E^{1}, E^{2}$ and $E^{3}$ provide a quantitative measurement of how much of the solution's energy is in the first, second and third streamwise harmonics, thus indicating whether the flowfield is likely to be dominated by singly, doubly or triply periodic vortices. Results in figure $20(b)$ show that at the pitchfork bifurcation points $R_{1}, R_{6}$ the solution is dominated by $E^{1}$, whilst at $R_{4}, R_{5}$ it is dominated by $E^{3}$, which confirms that the solution consists of pure $\mathrm{WTWI}^{1 / 1}$ and $\mathrm{wTWI}^{3 / 3}$ at these points, respectively. We do not find similar peaks in $E_{2}$ at $R_{2}$ or $R_{3}$, but using $E^{T}=E^{1}+E^{2}+E^{3}$, it is clear that the ratio $E^{2} / E^{T}$ is growing for $R_{3}<R o<R_{2}$ during the appearance of wTWI ${ }^{1 / 2}$. Between $R_{3}$ and $R_{4}$ both $E^{1} / E^{T}$ and $E^{2} / E^{T}$ become negligible, indicating the emergence of wTWI ${ }^{1 / 3}$.

To further explore the properties of the defected wTWI, in figure $21(a, b)$ we investigate bifurcations in the geometric parameter $\alpha_{s}$ for $R o=0.7$ and $R o=0.8$. The non-defected wTWI undergoes a supercritical pitchfork bifurcation towards $\mathrm{TVF}_{1}$ as 

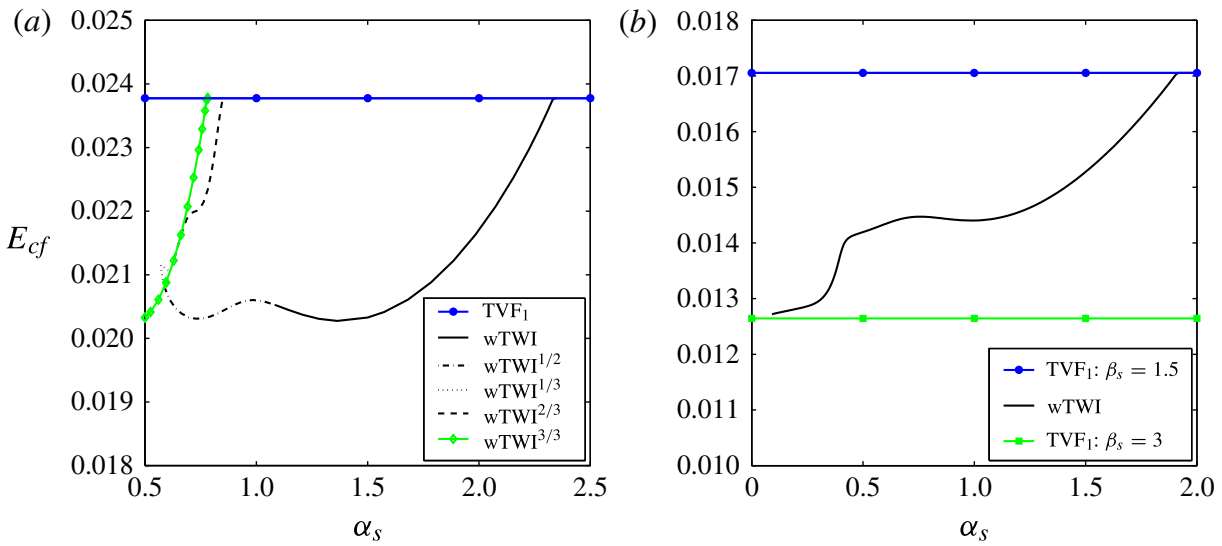

FIGURE 21. (Colour online) wTWI bifurcations in $\alpha_{s}$ with $\beta_{s}=1.5, \quad R e=100$. (a) Bifurcation in $\alpha_{s}$ with $R o=0.7$. Here, the solution encounters defected states at low $\alpha_{s}$, which prevent localization. (b) Bifurcation in $\alpha_{s}$ with $R o=0.8$. No defected states are passed, and wTWI bifurcates towards a streamwise-localized state of wavy twists separated by Taylor vortices with twice the original spanwise wavenumber, $\beta_{s}=3$.

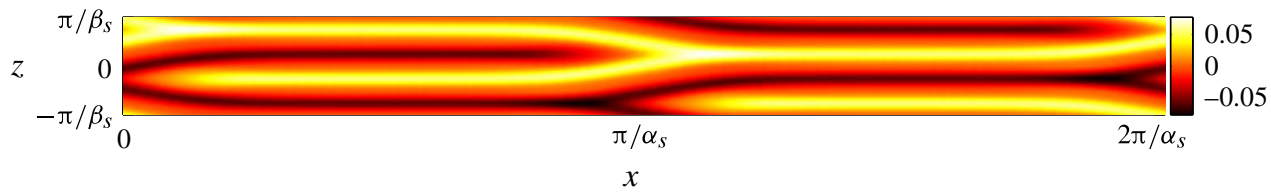

FIgURE 22. (Colour online) Streamwise-localized knotted wTWI with $\left(\alpha_{s}, \beta_{s}, R e, R o\right)=$ $(0.14,1.5,100,0.8)$. A colour plot of $u$ is shown in the $(x, z)$-plane at $y=0$.

$\alpha_{s}$ is increased, for both rotation numbers, but its behaviour at low $\alpha_{s}$ is different in each case. For $R o=0.7$ in figure 21(a), the defected solutions of figure 18 re-appear for $\alpha_{s}<0.95$. The continuation curve passes through wTWI ${ }^{1 / 2}, \mathrm{wTWI}^{1 / 3}$ and $\mathrm{wTWI}^{2 / 3}$ before re-connecting with $\mathrm{TVF}_{1}$. For $R o=0.8$ in figure $21(b)$ however, no wTWI ${ }^{i / j}$ states with $i<j$ are encountered, and the $\mathrm{wTWI}^{1 / 1}$ state begins a localization process, with a localized version of the twists, which we shall call knots, developing in the centre and at the edges of the streamwise-periodic domain as $\alpha_{s} \rightarrow 0$. The knots are separated by $\mathrm{TVF}_{1}$ arrangements with $\beta_{s}=3$, twice the spanwise wavenumber of the non-localized wTWI solution. A depiction of such a knotted vortex arrangement for $\alpha_{s}=0.14$ is given in figure 22. Given the proximity of the higher-harmonic wTWI solution branches for lower $R o$, we infer that streamwise localization is prevented at $R o=0.7$ due to the presence of the higher-harmonic wTWI states, which encourage the formation of $\mathrm{WTWI}^{i / j}$ solutions with $i<j$ rather than localization. As $\alpha_{s} \rightarrow 0$, the wTWI solution branch in figure $21(b)$ is shown to approach $\mathrm{TVF}_{1}$ with $\beta_{s}=3$, twice the spanwise wavenumber of the wTWI state. $\operatorname{TVF}_{1}\left(\beta_{s}=3\right)$ is stable to all wavenumber combinations for $(R e, R o)=(100,0.8)$; therefore, streamwise-localized WTWI may bifurcate in a subcritical, spanwise subharmonic bifurcation from $\operatorname{TVF}_{1}\left(\beta_{s}=3\right)$ with bifurcation point $\alpha_{s} \rightarrow 0$. 


\section{Comparison with experiments}

In this section we investigate the extent to which our stability analysis of secondary and tertiary states provides insight into the supercritical transitions observed in the experiments of Tsukahara et al. (2010) and Suryadi et al. (2014), who have mapped flow states in the $(\Omega, R e)$-plane. Our focus is on the observations of Suryadi et al. (2014), who charted the transition between laminar flow states for $R e=100$ and $\Omega=1.5,10,40$ and 90 , though we also include $(\Omega, R e)=(20,100)$ from Tsukahara et al. (2010). Laminar flow states, in this context, refer not only to the unique linear shear solution LAM, but to any stable higher-order bifurcation which does not exhibit chaotic dynamics.

Since we are concerned with the transition between laminar flow states, prior to transitions to quasi- or fully turbulent dynamics, our approach is to compute the number of attracting equilibria which bifurcate from secondary $\mathrm{TVF}_{1}$ states. We define a domain $\boldsymbol{D}$,

$$
\boldsymbol{D}=[0,2 \pi / 0.1] \times[-1,1] \times[0,2 \pi / 0.5],
$$

in the $x, y$ and $z$ directions, with fundamental wavenumbers $\alpha_{f}=0.1$ and $\beta_{f}=0.5$, chosen such that the aspect ratio of the domain is identical to the experimental $150 \mathrm{~h} \times$ $2 h \times 30 h$ domain used by Suryadi et al. (2014), though the full domain size is smaller so as to reduce to the number of states which we need to compute. We restrict our attention to the first three harmonics of the fundamental spanwise wavenumber, $\beta_{s}=$ $1,1.5,2$, and use the stability maps in figure 6 as a guide to determine the tertiary states which exist at streamwise wavenumbers harmonic to $\alpha_{f}=0.1$. We then use the Floquet theory techniques introduced in $\S 4$ to calculate the stability of the tertiary states to all wavenumber combinations supported by $\boldsymbol{D}$. If the state is stable to all such perturbations, then we interpret it as a stable attractor of $\boldsymbol{D}$. In every case considered, we find that there is more than one attracting state, meaning that there is a multiplicity of states in which the system could be at rest. It would be expected that a random perturbation could visit any of the attractor states.

Our results are summarized in table 2, where we chart the types of states observed at each $\Omega$ and the number of spatial configurations of each state (i.e. the $\left(\alpha_{s}, \beta_{s}\right)$ pairs for which it is stable in $\boldsymbol{D})$, alongside the experimental results of Tsukahara et al. (2010) and Suryadi et al. (2014). For $\Omega=1.5$ we have two stable $\mathrm{TVF}_{1}$ solutions with $\beta_{s}=1$ and $\beta_{s}=2$. This compares well with the experiment which records Cou2D, or streamwise-oriented roll cells. The spanwise wavelength of the roll cells given in Suryadi et al. (2014) is approximately $4.4 h$, which is close to $\beta_{s}=1.5$ with wavelength $2 \pi / 1.5=4.2 h$. However, we found that $\mathrm{TVF}_{1}$ with $\beta_{s}=1.5$ was marginally unstable, with an unstable eigenvalue $\sigma_{r}<1 \times 10^{-3}$. This unstable mode initiated a bifurcation towards a stable WVF state with $\alpha_{s}=0.3, \beta_{s}=1.5$.

The cases $\Omega=10$ and $\Omega=20$ are notable in that only one type of attractor state is found for each $\Omega$, though there are still different spatial scales over which the attractors are stable. In particular, the case $\left(\alpha_{s}, \beta_{s}\right)=(0.4,1.5)$ for $\Omega=10$ gives streamwise and spanwise wavelengths of approximately $15.7 \mathrm{~h}$ and $4.2 \mathrm{~h}$, which is in good agreement with the wavelengths of $15 \mathrm{~h}$ and $4 \mathrm{~h}$ given by Tsukahara et al. (2010) for Cou3D. For $\Omega=40$ we find eleven attractor states, the largest number for all the $\Omega$ cases which we considered. Most of these are TWI states, which is in agreement with the observations of Suryadi et al. (2014) who report twisted roll cells for $\Omega=$ $40,52,70$.

The experiments for $\Omega=90$ had the interesting outcome that two distinct stable flow visualizations were found, depending upon the initial conditions at the beginning 
$\Omega \quad R o=\Omega / R e$

$\begin{array}{clcc} & & \text { State } & \text { Cardinality } \\ 1.5 & 0.015 & \mathrm{TVF}_{1} & 2 \\ & & \text { WVF } & 1 \\ 10 & 0.1 & \text { WVF } & 4 \\ & & & \\ 20 & 0.2 & \text { TVF }_{1} & 3 \\ 40 & 0.4 & \text { TWI }^{2} & 9 \\ & & \text { TVF }_{1} & 2 \\ 90 & 0.9 & \text { wTWI }^{2} & 4\end{array}$

$\mathrm{TVF}_{1}$
Attracting equilibria

$\left(\alpha_{s}, \beta_{s}\right)$

$\begin{array}{cc}(0,1),(0,2) & \text { Cou2D } \\ (0.3,1.5) & \\ (0.3,1),(0.4,1) & \text { Cou3D } \\ (0.4,1.5),(0.5,1.5) & \\ (0,1),(0,1.5),(0,2) & \text { Cou2Dh } \\ (1.1,1), \ldots,(1.9,1) & \text { Twist } \\ (0,1.5),(0,2) & \\ (0.3,1) & \text { Undetermined } \\ (0.5,1.5), \ldots,(0.7,1.5) & \\ (0,2) & \end{array}$

TABLE 2. The attracting equilibrium states of the domain $\boldsymbol{D}=[0,2 \pi / 0.1] \times[-1,1] \times$ $[0,2 \pi / 0.5]$ for $R e=100$ and various $\Omega$. For each $\Omega$ and corresponding $R o$ we list the types of stable equilibria we have computed, the cardinality of each type (i.e. the number of different wavenumber configurations at which it is stable), and the specific stable wavenumber pairs for each state. In the final column we list the results of experimental investigations for corresponding parameters. Cou2D $\equiv$ streamwise-oriented roll cells. Cou3D $\equiv$ wavy streamwise-oriented roll cells. Cou2Dh $\equiv$ streamwise oriented roll cells at higher rotation numbers. Twist $\equiv$ twisted roll cells. Undetermined $\equiv$ two distinct flow visualizations are recorded (see Suryadi et al. 2014).

of the experiment. The two observed flowfields are re-printed in figure 23(a,c). Figure 23(a) has been likened to the braided vortices observed by Andereck et al. (1983), while the staggered pattern of figure 23(c) is unidentified. In our table of attractor states we find four wTWI solutions at various $\left(\alpha_{s}, \beta_{s}\right)$ combinations, and in figure $23(d)$ we plot the stable wTWI state with $\left(\alpha_{s}, \beta_{s}\right)=(0.7,1.5)$ by way of comparison with the staggered flowfield. Though there remains some discrepancy between the spatial scales of the structures in figure $23(c, d)$, we posit that the qualitative staggered nature of the experimentally observed flowfield is well captured by the wTWI configuration. To further investigate the braided vortex flowfield in figure 23(a), we conducted a large periodic-domain simulation of a randomly generated initial condition comprised of Stokes modes, on a domain $\boldsymbol{D}_{2}$,

$$
\boldsymbol{D}_{2}=[0,2 \pi / 0.08] \times[-1,1] \times[0,2 \pi / 0.4],
$$

which is slightly larger than $\boldsymbol{D}$, but with an equivalent aspect ratio. The simulation approached a steady state with a vortex localized in both the streamwise and spanwise directions around streamwise-independent Taylor vortices. This was then used as a guess in the Newton procedure of $\S 3$ to determine a new stable equilibrium solution, with the localized vortex feature retained. The flowfield of the solution is depicted in figure 24. We note the similarity of the flowfield with the knotted wTWI solution in figure 22, from which we infer that our solution is a streamwise and spanwise localized, or knotted, wTWI. The knot features of this solution could also be analysed in terms of the defects observed in Taylor-Couette and Taylor-Dean systems as discussed in Bot \& Mutabazi (2000), Nana, Ezersky \& Mutabazi (2009), Ezersky, Abcha \& Mutabazi (2010) and Abcha et al. (2013). Nana et al. (2009) and Ezersky et al. (2010) use a complex Ginsburg-Landau equation to model defects of spiral 
(a)

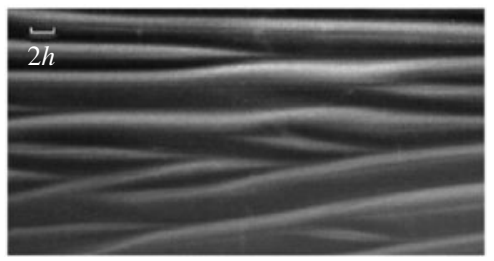

(c)

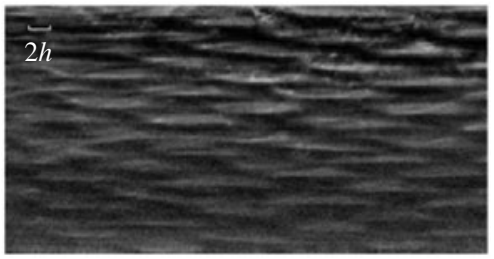

(b)

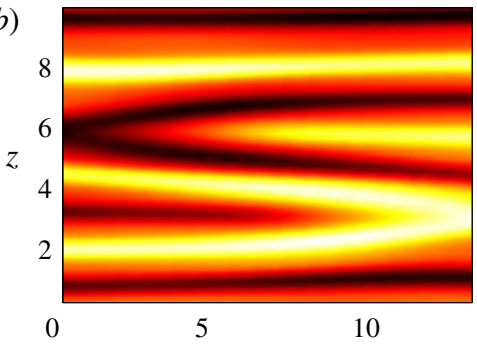

(d)

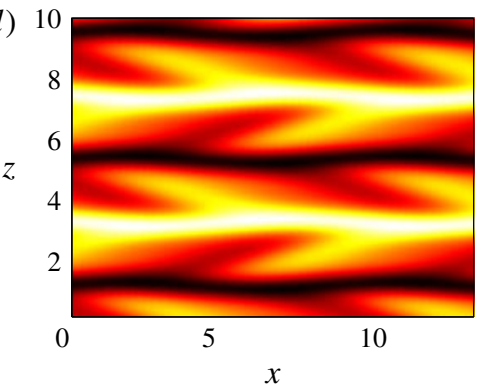

FIgURE 23. (Colour online) Comparison between computational attractor states and experimentally observed flow structures for $(\Omega, R e)=(90,100)$. The computational flowfields $(b)$ and $(d)$ are colour plots of $u$ in the $(x, z)$-plane at the wall-normal mid-point $y=0$. (a) First experimentally observed flowfield, (b) BVF on a reduced domain, (c) 'staggered' flowfield pattern, $(d)$ wTWI state with $\left(\alpha_{s}, \beta_{s}\right)=(0.7,1.5)$. Suryadi et al. (2014) state that the effective flowfield photograph size of $(a)$ and $(c)$ is approximately $13.6 h \times 10 h$ in the streamwise and spanwise directions. We therefore reduce the domain sizes to an appropriate length for figures $(b)$ and $(d)$.

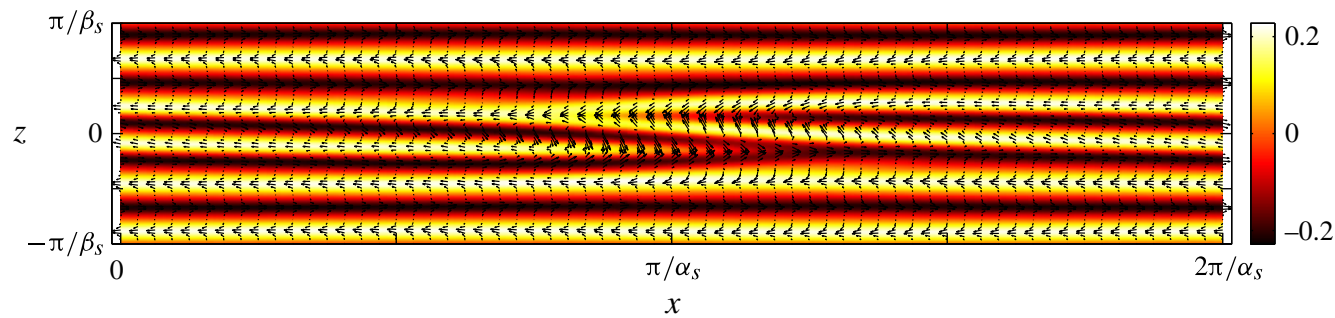

FIgURE 24. (Colour online) Braided vortex flow (BVF) with $\left(\alpha_{s}, \beta_{s}, R e, R o\right)=$ $(0.08,0.4,100,0.9)$. Stable equilibrium solution found by simulation of a random perturbation. The flowfield contains a localized fold in its centre, and is depicted here with a colour plot of $v$ and a vector plot of $(u, w)$ at the mid-plane $y=0$. The name BVF is in reference to the similarity of the flowfield to the braided vortices observed by Andereck, Dickman \& Swinney (1983) and Suryadi et al. (2014).

solutions in Taylor-Couette flow, and it is plausible that the production of knots in our localized solution could be a result of the same mechanism, though we do not pursue evidence for this here. In figure 23(b), we plot a close up of the knotted region of the localized wTWI state alongside the braided vortex flowfield observed experimentally. The flow features compare well, and indeed the spatial scales appear consistent. We therefore name the localized wTWI state braided vortex flow (BVF). 
Our simple modelling approach to experimental transition is successful in accounting for the observed qualitative flow features, insofar as the experimental flow states can be identified with secondary or tertiary states of RPCF. Despite working on a smaller domain than is used in the experiments, some of the stable states we have attained provide reasonable agreement with the streamwise and spanwise wavelengths of physically observed structures. However, as noted by Tsukahara et al. (2010), Ekman layers are likely to form at the lower boundary of their apparatus, causing a change in wavelengths between structures near the lower wall and those away from it, as discussed by Czarny et al. (2003), Hollerbach \& Fournier (2004), Altmeyer et al. (2010) and Heise et al. (2013). It may therefore be necessary to include the effects of Ekman layers should further agreement between experiment and theory be sought.

\section{Summary and conclusions}

In this paper we have studied instability and transition in supercritical RPCF. In $\S 6$ we performed an extensive Floquet stability analysis on $\mathrm{TVF}_{1}$, at Reynolds numbers identified to be in the transitional flow regime by the experimental study of Tsukahara et al. (2010). The stability properties of $\mathrm{TVF}_{1}$ are shown to be dependent on $R e$, $R o$ and the wavenumber of the vortex. Instabilities grow in range and magnitude as $R e$ is increased, giving an indication of a wide range of tertiary instabilities possible over the transition region. Tertiary states which bifurcate from $\mathrm{TVF}_{1}$ are presented in terms of their bifurcation and stability behaviour in $\S 7$. With focus on variation in $R o$, we study the bifurcation behaviour of the tertiary periodic orbit oWVF in $\S 8$. Three-dimensional bifurcation diagrams reveal the emergence of an unstable attractor as oWVF bifurcates from $\mathrm{TVF}_{1}$, wherein a homoclinic tangle appears to occur about an unstable oWVF state. In $\S 9$ we introduce a new streamwise-independent tertiary flow state, SVF, which breaks the mirror symmetry $\boldsymbol{Z}$ of Taylor vortices without losing two-dimensionality. Furthermore, as it bifurcates in Ro SVF is found to connect the first and second Taylor vortex solutions. In our bifurcation analysis of wTWI in $\S 10$, wTWI states are found to develop flowfield defects at low $\alpha_{s}$, and we present evidence that the defects prevent streamwise localization at moderate rotation numbers.

Turning to physical experiments, in $\S 11$ we explored the extent to which the secondary and tertiary structures from $\S 6$ and $\S 7$ can be used to describe the flow regimes observed in the works of Tsukahara et al. (2010) and Suryadi et al. (2014). We calculated the different stable attractor states which co-exist at a range of experimentally relevant parameter values, which we are then able to compare with experimentally visualized flow states. Our simulations yielded an equilibrium state which consists of a streamwise and spanwise localized fold amongst an array of streamwise vortices which we have named BVF, for its similarity to the braided vortex structures observed both by Andereck et al. (1983) and Suryadi et al. (2014).

Further work on RPCF should investigate the routes to chaos that are supported in the system, and the exact flow states involved in the transition to chaos should be compared with the coherent structures observed in physical experiments. This would serve to enhance our understanding of supercritical transition, and underscore the efficacy of the dynamical systems approach to the transition to turbulence.

\section{Acknowledgements}

C.A.D. would like to thank Dr A. Suryadi for sharing experimental results and Dr E. J. Brambley for many helpful discussions towards this work. C.A.D. gratefully 
acknowledges EPSRC PhD studentship funding. The authors would also like to thank the journal referees, whose careful reading of the manuscript provided important insights into the material.

\section{Appendix A}

As discussed by Gibson et al. (2009), the equations of non-rotating PCF satisfy the discrete dihedral group $D_{1} \times D_{1}=\left\{e, \sigma_{x}, \sigma_{z}, \sigma_{x z}\right\}$, where $e$ is the identity operation and $\sigma_{x}, \sigma_{z}$ and $\sigma_{x z}=\sigma_{x} \sigma_{z}$ are the rotation operations

$$
\begin{gathered}
\sigma_{x}[u, v, w](x, y, z)=[-u,-v, w](-x,-y, z), \\
\sigma_{z}[u, v, w](x, y, z)=[u, v,-w](x, y,-z), \\
\sigma_{x z}[u, v, w](x, y, z)=[-u,-v,-w](-x,-y,-z) .
\end{gathered}
$$

Since there are two homogeneous directions in PCF, the equations also satisfy the $S O(2) \times S O(2)$ continuous two-parameter group of streamwise-spanwise translations

$$
\tau\left(\ell_{x}, \ell_{z}\right)[u, v, w](x, y, z)=[u, v, w]\left(x+\ell_{x}, y, z+\ell_{z}\right) .
$$

Combining rotation and translation, the equations of PCF are equivariant under the symmetry group $\Gamma=O(2)_{x} \times O(2)_{z}=D_{1, x} \ltimes S O(2) \times D_{1, z} z \ltimes S O(2)$, where $\ltimes$ is the semi-direct product, the subscript $x$ indicates streamwise translations and sign changes in $x$ and $y$, and the subscript $z$ indicates spanwise translations and sign changes in $z$.

We show here that the addition of Coriolis rotation about the spanwise axis breaks none of these symmetries, thus the equations of RPCF are also equivariant under $\Gamma$. Clearly, spanwise rotation of RPCF does not alter the translation invariance of PCF in the streamwise and spanwise directions. Thus, it simply remains to be shown that RPCF is invariant under $\sigma_{x}$ and $\sigma_{z}$, since $\sigma_{x z}=\sigma_{x} \sigma_{z}$. Moreover, we need only be concerned with the $x$ and $y$ momentum equations, since these are the only ones to contain Coriolis forcing terms. Using $\widetilde{L}_{N S}$ to denote the non-rotating nonlinear NavierStokes operator, we have

$$
\begin{aligned}
& \sigma_{x}\left\{\frac{\partial u}{\partial t}-\boldsymbol{i} \cdot \widetilde{L}_{N S} \boldsymbol{u}-\operatorname{Rov}\right\}=-\left(\frac{\partial u}{\partial t}-\boldsymbol{i} \cdot \widetilde{L}_{N S} \boldsymbol{u}-\operatorname{Rov}\right)=0, \\
& \sigma_{x}\left\{\frac{\partial v}{\partial t}-\boldsymbol{j} \cdot \widetilde{L}_{N S} \boldsymbol{u}+\operatorname{Rou}\right\}=-\left(\frac{\partial v}{\partial t}-\boldsymbol{j} \cdot \widetilde{L}_{N S} \boldsymbol{u}+\operatorname{Rou}\right)=0
\end{aligned}
$$

hence

$$
\sigma_{x}\left\{\frac{\partial \boldsymbol{u}}{\partial t}-\widetilde{L}_{N S} \boldsymbol{u}-R o(\boldsymbol{u} \times \boldsymbol{k})\right\}=\frac{\partial \boldsymbol{u}}{\partial t}-\widetilde{L}_{N S} \boldsymbol{u}-\operatorname{Ro}(\boldsymbol{u} \times \boldsymbol{k})=0 .
$$

Similarly, for $\sigma_{z}$ we have

$$
\sigma_{z}\left\{\frac{\partial \boldsymbol{u}}{\partial t}-\widetilde{L}_{N S} \boldsymbol{u}-\operatorname{Ro}(\boldsymbol{u} \times \boldsymbol{k})\right\}=\frac{\partial \boldsymbol{u}}{\partial t}-\widetilde{L}_{N S} \boldsymbol{u}-\operatorname{Ro}(\boldsymbol{u} \times \boldsymbol{k})=0 .
$$

We have therefore shown that RPCF, like PCF, satisfies the dihedral symmetry group $D_{1} \times D_{1}=\left\{e, \sigma_{x}, \sigma_{z}, \sigma_{x, z}\right\}$ and the $O(2)_{x} \times O(2)_{z}$ group $\Gamma$. We find no indication that the Coriolis terms place any further symmetry restrictions on the RPCF system, relative to the PCF symmetries. 


\section{Appendix B}

Using the relations

$$
\alpha_{m}=\alpha+m \alpha_{s}, \quad \beta_{n}=\beta+n \beta_{s}, \quad k_{m n}^{2}=\alpha_{m}^{2}+\beta_{n}^{2},
$$

we define the operators $P_{m n}, Q_{m n}$ and $F_{m n}^{j k}$ from (4.13) as follows:

$$
\begin{gathered}
P_{m n}=\left(\begin{array}{cc}
\left(D^{2}-k_{m n}^{2}\right) k_{m n}^{2} & 0 \\
0 & k_{m n}^{2}
\end{array}\right), \\
Q_{m n}=\left(\begin{array}{cc}
Q^{11} & Q^{12} \\
Q^{21} & Q^{22}
\end{array}\right),
\end{gathered}
$$

where we have

$$
\begin{aligned}
& Q^{11}= k_{m n}^{2}\left\{\frac{1}{R e}\left(k_{m n}^{4}-2 k_{m n}^{2} D^{2}+D^{4}\right)+\mathrm{i} \alpha_{m} U\left(k_{m n}^{2}-D^{2}\right)\right. \\
&\left.+\mathrm{i} \beta_{n} W\left(k_{m n}^{2}-D^{2}\right)+\mathrm{i} \alpha_{m} U^{\prime \prime}+\mathrm{i} \beta_{n} W^{\prime \prime}\right\}, \\
& Q^{12}=-k_{m n}^{2} \mathrm{i} \beta_{n} R o, \\
& Q^{21}= k_{m n}^{2}\left\{\mathrm{i} \beta_{n} R o-\mathrm{i} \beta_{n} U^{\prime}-\mathrm{i} \alpha_{m} W^{\prime}\right\}, \\
& Q^{22}= k_{m n}^{2}\left\{\frac{1}{R e}\left(k_{m n}^{2}-D^{2}\right)+\mathrm{i} \alpha_{m} U+\mathrm{i} \beta_{n} W\right\} ; \\
& F_{m n}^{j k}=G_{m n}^{j k} H_{m n}^{j k},
\end{aligned}
$$

with

$$
\begin{gathered}
G_{m n}^{j k}=\left(\begin{array}{ccc}
\mathrm{i} j \alpha_{s} U_{j k}+\mathscr{F} & D U_{j k} & \mathrm{i} k \beta_{s} U_{j k} \\
\mathrm{ij} \alpha_{s} V_{j k} & D V_{j k}+\mathscr{F} & \mathrm{i} k \beta_{s} V_{j k} \\
\mathrm{i} j \alpha_{s} W_{j k} & D W_{j k} & \mathrm{i} k \beta_{s} W_{j k}+\mathscr{F}
\end{array}\right), \\
H_{m n}^{j k}=\left(\begin{array}{cc}
\mathrm{i} \alpha_{m-j} D & -\mathrm{i} \beta_{n-k} \\
\alpha_{m-j}^{2}+\beta_{n-k}^{2} & 0 \\
\mathrm{i} \beta_{n-k} D & \mathrm{i} \alpha_{m-j}
\end{array}\right), \\
\mathscr{F}=\mathrm{i} \alpha_{m-j} U_{j k}+V_{j k} D+\mathrm{i} \beta_{n-k} W_{j k}, \\
U_{j k}=\left(U_{j k}, V_{j k}, W_{j k}\right) .
\end{gathered}
$$

\section{REFERENCES}

Abcha, N., Crumeyrolle, O., Ezersky, A. B. \& Mutabazi, I. 2013 Velocity field of the spiral vortex flow in the Couette-Taylor system. Eur. Phys. J. E 36 (3), 20.

Abshagen, J., Lopez, J. M., Marques, F. \& Pfister, G. 2005 Symmetry breaking via global bifurcations of modulated rotating waves in hydrodynamics. Phys. Rev. Lett. 94 (7), 074501.

Alfredsson, P. H. \& Tillmark, N. 2005 Instability, transition and turbulence in plane Couette flow with system rotation. In IUTAM Symposium on Laminar Turbulent Transition and Finite Amplitude Solutions (ed. T. Mullin \& R. R. Kerswell), Fluid Mechanics and its Applications, vol. 77. Springer. 
Altmeyer, S., Hoffmann, Ch., Heise, M., Abshagen, J., Pinter, A., Lücke, M. \& Pfister, G. 2010 End wall effects on the transition between Taylor vortices and spiral vortices. Phys. Rev. E 81 (6), 066313.

Andereck, C. D., Dickman, R. \& Swinney, H. L. 1983 New flows in a circular Couette system with co-rotating cylinders. Phys. Fluids 26 (6), 1395-1401.

Andereck, C. D., LiU, S. S. \& Swinney, H. L. 1986 Flow regimes in a circular Couette system with independently rotating cylinders. J. Fluid Mech. 164, 155-183.

AntonijoAn, J. \& SÁnchez, J. 2000 Transitions from Taylor vortex flow in a co-rotating TaylorCouette system. Phys. Fluids 12 (12), 3147-3159.

Benjamin, T. B. \& Mullin, T. 1982 Notes on the multiplicity of flows in the Taylor experiment. J. Fluid Mech. 121, 219-230.

Bot, P. \& Mutabazi, I. 2000 Dynamics of spatio-temporal defects in the Taylor-Dean system. Eur. Phys. J. B 13, 141-155.

Clever, R. M. \& Busse, F. H. 1997 Tertiary and quaternary solutions for plane Couette flow. J. Fluid Mech. 344, 137-153.

Coles, D. 1965 Transition in circular Couette flow. J. Fluid Mech. 21, 385-425.

Czarny, O., Serre, E., Bontoux, P. \& Lueptow, R. M. 2003 Interaction between Ekman pumping and the centrifugal instability in Taylor-Couette flow. Phys. Fluids 15 (2), 467-477.

Davey, A., DiPrima, R. C. \& Stuart, J. T. 1968 On the instability of Taylor vortices. J. Fluid Mech. 31, 17-52.

Deguchi, K. \& Altmeyer, S. 2013 Fully nonlinear mode competitions of nearly bicritical spiral or Taylor vortices in Taylor-Couette flow. Phys. Rev. E 87 (4), 043017.

Duguet, Y., Pringle, C. T. \& Kerswell, R. R. 2008 Relative periodic orbits in transitional pipe flow. Phys. Fluids 20 (11), 114102.

Eagles, P. M. 1971 On stability of Taylor vortices by fifth-order amplitude expansions. J. Fluid Mech. 49, 529-550.

Eckhaus, W. 1965 Studies in Nonlinear Stability Theory. Springer.

Ezersky, A. B., АвChA, N. \& Mutabazi, I. 2010 The structure of spatio-temporal defects in a spiral pattern in the Couette-Taylor flow. Phys. Lett. A 37 (33), 3297-3303.

Faisst, H. \& Eckhardt, B. 2003 Travelling waves in pipe flow. Phys. Rev. Lett. 91 (22), 224502.

Gibson, J. F. 2008 Channelflow: a spectral Navier-Stokes simulator in C++. Tech. Rep. Georgia Institute of Technology.

Gibson, J. F., Halcrow, J. \& Cvitanović, P. 2008 Visualizing the geometry of state-space in plane Couette flow. J. Fluid Mech. 611, 107-130.

Gibson, J. F., Halcrow, J. \& Cvitanović, P. 2009 Equilibrium and travelling-wave solutions of plane Couette flow. J. Fluid Mech. 683, 243-266.

Hegseth, J. J., Baxter, G. W. \& Andereck, C. D. 1996 Bifurcations from Taylor vortices between corotating concentric cylinders. Phys. Rev. E 53, 507-521.

Heise, M., Hoffmann, Ch., Will, Ch., Altmeyer, S., Abshagen, J. \& Pfister, G. 2013 Co-rotating Taylor-Couette flow enclosed by stationary disks. J. Fluid Mech. 716, 507-521.

Hiwatashi, K., Alfredsson, P. H., Tillmark, N. \& Nagata, M. 2007 Experimental observations of instabilities in rotating plane couette flow. Phys. Fluids 19, 48-103.

Hollerbach, R. \& Fournier, A. 2004 End-effects in a rapidly rotating cylindrical Taylor-Couette flow. In MHD Couette Flows: Experiments and Models (ed. R. Rosner, G. Rüdiger \& A. Bonanno), vol. 733, pp. 114-121. AIP Conf. Proc.

Itano, T. \& Generalis, S. C. 2009 Hairpin vortex solution in planar Couette flow: a tapestry of knotted vortices. Phys. Rev. Lett. 102, 114-501.

Kawahara, G. \& KidA, S. 2001 Periodic motion embedded in plane Couette turbulence: regeneration cycle and burst. J. Fluid Mech. 449, 291-300.

Koschmieder, E. L. 1993 Bénard Cells and Taylor Vortices. Cambridge University Press.

Kreilos, T. \& ECKhardT, B. 2012 Periodic orbits near the onset of chaos in plane Couette flow. Chaos 22 (4), 047505. 
Lezius, D. K. \& Johnston, J. P. 1976 Roll-cell instabilities in rotating laminar and turbulent channel flows. J. Fluid Mech. 77, 153-175.

Lopez, J. M. \& MARqueS, F. 2005 Finite aspect ratio Taylor-Couette flow: Shil'nikov dynamics of 2-tori. Physica D 211, 168-191.

Mellibovsky, F. \& ECKhARDt, B. 2012 From travelling waves to mild chaos: a supercritical bifurcation cascade in pipe flow. J. Fluid Mech. 709, 149-190.

Mullin, T. 2010 The rich structure of transition in a shear flow. J. Fluid Mech. 648, 1-4.

NagatA, M. 1986 Bifurcations in Couette flow between almost corotating cylinders. J. Fluid Mech. 169, 229-250.

NagATA, M. 1988 On wavy instabilities of the Taylor-vortex flow between corotating cylinders. J. Fluid Mech. 188, 585-598.

NagATA, M. 1990 Three-dimensional finite-amplitude solutions in plane Couette flow: bifurcation from infinity. J. Fluid Mech. 217, 519-527.

NagAtA, M. 1997 Three-dimensional travelling-wave solutions in plane Couette flow. Phys. Rev. E 55, 2023-2025.

NagAtA, M. 1998 Tertiary solutions and their stability in rotating plane Couette flow. J. Fluid Mech. 358, 357-378.

NagAtA, M. 2013 A note on the mirror-symmetric coherent structure in plane Couette flow. J. Fluid Mech. 727, R1.

NAGATA, M. \& KaWAhaRA, G. 2004 Three-dimensional periodic solutions in rotating/non-rotating plane Couette flow. In Advances in Turbulence X, Proceedings of the 10th European Turbulence Conference (ed. H. I. Andersson \& P. A. Krogstad), vol. 77. CIMNE.

NAnA, L., Ezersky, A. B. \& MutabaZI, I. 2009 Secondary structures in a one-dimensional complex Ginzburg-Landau equation with homogeneous boundary conditions. Proc. R. Soc. Lond. A 465, 2251-2265.

Pringle, C. T. \& Kerswell, R. R. 2007 Asymmetric, helical, and mirror-symmetric travelling waves in pipe flow. Phys. Rev. Lett. 99 (7), 074502.

Schmid, P. J. \& Henningson, D. S. 2001 Stability and Transition in Shear Flows. Springer.

Schneider, T. M., Gibson, J. F., LAhgA, M., De Lillo, F. \& EcKhardt, B. 2008 Laminar turbulent boundary in plane Couette flow. Phys. Rev. E 78.

Shilnikov, L. P. 1965 A case of the existence of a denumerable set of periodic motions. Sov. Math. Dokl. 6, 163-166.

Suryadi, A., Segalini, A. \& Alfredsson, P. H. 2014 Zero absolute vorticity: insight from experiments in rotating laminar plane Couette flow. Phys. Rev. E 89 (3), 033003.

Suryadi, A., Tillmark, N. \& Alfredsson, P. H. 2013 Velocity measurements of streamwise roll cells in rotating plane Couette flow. Exp. Fluids 15 (11), 1617.

TAYLOR, G. I. 1923 Stability of a viscous liquid contained between two rotating cylinders. Phil. Trans. R. Soc. Lond. 223, 289-343.

Tsukahara, T., Tillmark, N. \& Alfredsson, P. H. 2010 Flow regimes in plane Couette flow with system rotation. J. Fluid Mech. 648, 5-33.

Viswanath, D. 2007 Recurrent motions within plane Couette turbulence. J. Fluid Mech. 580, 339-358.

Waleffe, F. 1997 On a self-sustaining process in shear flows. Phys. Fluids 9, 883-900.

WALeffe, F. 1998 Three-dimensional coherent states in plane shear flows. Phys. Rev. Lett. 81, $4140-4143$.

WALEFfe, F. 2003 Homotopy of exact coherent structures in plane shear flows. Phys. Fluids 15, $1517-1534$.

WANG, J., GibSon, J. F. \& WALEFFe, F. 2007 Lower branch coherent states in shear flows: transition and control. Phys. Rev. Lett. 98 (20), 204501.

Wedin, H. \& Kerswell, R. R. 2004 Exact coherent structures in pipe flow: travelling wave solutions. J. Fluid Mech. 508, 333-371.

WeisshaAR, E., Busse, F. H. \& Nagata, M. 1991 Twist vortices and their instabilities in the Taylor-Couette system. J. Fluid Mech. 226, 549-564.

Wiggins, S. 2003 Introduction to Applied Nonlinear Dynamical Systems and Chaos. Springer. 